\title{
SOME INEQUALITIES IN THE THEORY OF FUNCTIONS $\left({ }^{1}\right)$
}

\section{BY \\ ZEEV NEHARI}

1. Introduction. Many of the inequalities of function theory and potential theory may be reduced to statements regarding the properties of harmonic domain functions with vanishing or constant boundary values, that is, functions which can be obtained from the Green's function by means of elementary processes. For the derivation of these inequalities a large number of different techniques and procedures have been used. It is the aim of this paper to show that many of the known inequalities of this type, and also others which are new, can be obtained as simple consequences of the classical minimum property of the Dirichlet integral. In addition to the resulting simplification, this method has the further advantage of being capable of generalization to a wide class of linear partial differential equations of elliptic type in two or more variables.

The idea of using the positive-definite character of an integral as the point of departure for the derivation of function-theoretic inequalities is, of course, not new and it has been successfully used for this purpose by a number of authors $[1 ; 2 ; 8 ; 9 ; 16]$. What the present paper attempts is to give a more or less systematic survey of the type of inequality obtainable in this way.

2. Monotonic functionals. 1 . The domains we shall consider will be assumed to be bounded by a finite number of closed analytic curves and they will be embedded in a given closed Riemann surface $R$ of finite genus. The symbol $S(z)$ will be used to denote a "singularity function" with the following properties: $S(z)$ is real, harmonic, and single-valued on $R$, with the exception of a finite number of points at which $S(z)$ has specified singularities. $S(z)$ is thus the real part of a properly normalized Abelian integral.

The following result indicates a monotonic functional associated with $S(z)$.

Theorem I. Let $D$ and $D_{1}$ be two domains embedded in $R$ such that $D \subset D_{1}$ and that $D_{1}-D$ contains no singularities of $S(z)$, and let $C$ and $C_{1}$ denote the boundaries of $D$ and $D_{1}$ respectively. Let further $p(z)$ denote the function which vanishes on $C$ and is such that $p(z)+S(z)$ is harmonic in $D$. If $p_{1}(z)$ denotes the corresponding function associated with $D_{1}$, then

$$
\int_{C} S(z) \frac{\partial p(z)}{\partial n} d s \geqq \int_{C_{1}} S(z) \frac{\partial p_{1}(z)}{\partial n} d s,
$$

Presented to the Society, September 5, 1952; received by the editors May 29, 1952.

(1) Work supported by the Office of Naval Research under Contract N6ori-106 Task Order 5 (NR-043-992). 
where the differentiation is performed with respect to the outer normal.

With the notation $(u, u)_{D}=\iint_{D}\left(u_{x}^{2}+u_{y}^{2}\right) d x d y$, it follows from the minimum property of the Dirichlet integral that

$$
\left(p_{1}+S, p_{1}+S\right)_{D_{1}} \leqq(u, u)_{D_{1}} \text {, }
$$

where $u(z)$ is continuous in the closure of $D_{1}$ and has on $C_{1}$ the same boundary values as $p_{1}(z)+S(z)$; the derivatives $u_{x}$ and $u_{y}$ need only be continuous in subdomains of $D_{1}$ into which $D_{1}$ is divided by a finite number of smooth arcs or curves. Since $p(z)$ and $p_{1}(z)$ vanish on $C$ and $C_{1}$, respectively, we may set $u(z)=p(z)+S(z)$ in $D$ and $u(z)=S(z)$ in $D_{1}-D$. Inserting this in (2) and using Green's formula, we obtain

$$
\int_{C_{1}} S\left(\frac{\partial S}{\partial n}+\frac{\partial p_{1}}{\partial n}\right) d s \leqq \int_{C} S\left(\frac{\partial S}{\partial n}+\frac{\partial p}{\partial n}\right) d s+\int_{C_{1}} S \frac{\partial S}{\partial n} d s-\int_{C} S \frac{\partial S}{\partial n} d s .
$$

This is identical with (1) and thus proves Theorem I.

REMARK. From the proof it is clear that the assumptions regarding the single-valuedness of the various functions involved may be somewhat relaxed. It is sufficient to assume that $S(z)$ is single-valued in $D_{1}-D$, while $S(z)+p(z)$ and $S(z)+p_{1}(z)$ are single-valued in $D$ and $D_{1}$, respectively.

2. To illustrate the application of Theorem $I$, consider the case in which $R$ is the schlicht plane and $D$ is a simply-connected domain. If $\alpha_{1}, \cdots, \alpha_{n}$ are complex constants such that $\alpha_{1}+\cdots+\alpha_{n}=0, \zeta_{1}, \cdots, \zeta_{n}$ are points of $D$, and $w=F(z)$ is one of the functions mapping $D$ onto the schlicht unit circle, it is easily confirmed that

$$
p(z)=\operatorname{Re}\left\{\sum_{\nu=1}^{n}\left[\alpha_{\nu} \log \left(F(z)-F\left(\zeta_{\nu}\right)\right)-\alpha_{\nu}^{*} \log \left(1-F^{*}\left(\zeta_{\nu}\right) F(z)\right)\right]\right\}\left({ }^{2}\right)
$$

vanishes on $C$. The corresponding singularity function $S(z)$ is of the form

$$
S(z)=-\operatorname{Re}\left\{\sum_{\nu=1}^{n} \alpha_{\nu} \log \left(z-\zeta_{\nu}\right)\right\}
$$

Clearly, $p(z)+S(z)$ is single-valued in $D$ and-because of $\sum_{v=1}^{n} \alpha_{\nu}=0-S(z)$ is single-valued in the complement of $D$. In view of the remark made further above, Theorem I will therefore apply. The integral in (1) can be evaluated by the residue theorem. If $q(z)$ and $\sigma(z)$ are the analytic functions for which $p(z)=\operatorname{Re}\{q(z)\}$ and $S(z)=\operatorname{Re}\{\sigma(z)\}$, we have, in view of $p(z)=0(z \in C)$,

$$
\frac{\partial p}{\partial n} d s=\frac{1}{i} q^{\prime}(z) d z
$$$$
z \in C
$$

and therefore

(2) Asterisks denote conjugate complex quantities. 


$$
\begin{aligned}
\int_{C} S \frac{\partial p}{\partial n} d s=\int_{C}(S+p) \frac{\partial p}{\partial n} d s & =\operatorname{Re}\left\{\int_{C}(\sigma(z)+q(z)) \frac{\partial p}{\partial n} d s\right\} \\
& =\operatorname{Re}\left\{\frac{1}{i} \int_{C}(\sigma(z)+q(z)) q^{\prime}(z) d z\right\} .
\end{aligned}
$$

Since $q(z)+\sigma(z)$ is regular in $D$, this is equivalent to

$$
\int_{C} S \frac{\partial p}{\partial n} d s=-\operatorname{Re}\left\{\frac{1}{i} \int_{C}(\sigma(z)+q(z)) \sigma^{\prime}(z) d z\right\} .
$$

Inserting the expressions for $\sigma(z)$ and $q(z)$, we obtain

$$
\begin{aligned}
\int_{C} S \frac{\partial p}{\partial n} d s=\operatorname{Re}\left\{\frac { 1 } { i } \int _ { C } \left(\sum _ { \nu = 1 } ^ { n } \left[\alpha_{\nu} \log \frac{F(z)-F\left(\zeta_{\nu}\right)}{z-\zeta_{\nu}}\right.\right.\right. \\
\left.\left.\left.-\alpha_{\nu}^{*} \log \left(1-F^{*}\left(\zeta_{\nu}\right) F(z)\right)\right]\right)\left(\sum_{\mu=1}^{n} \frac{\alpha_{\mu}}{z-\zeta_{\mu}}\right) d z\right\} \\
=2 \pi \operatorname{Re}\left\{\sum_{\nu, \mu=1}^{n} \alpha_{\nu} \alpha_{\mu} \log \frac{F\left(\zeta_{\nu}\right)-F\left(\zeta_{\mu}\right)}{\zeta_{\nu}-\zeta_{\mu}}\right. \\
\left.-\sum_{\nu, \mu=1}^{n} \alpha_{\nu}^{*} \alpha_{\mu} \log \left(1-F^{*}\left(\zeta_{\nu}\right) F\left(\zeta_{\mu}\right)\right)\right\} .
\end{aligned}
$$

By Theorem I, this expression decreases if the domain $D$ increases. If $D$ is contained in the unit circle, we may compute the right-hand side of (1) by replacing, in (5), $F(z)$ by $z$. (1) and (5) will then yield the inequality

$$
\operatorname{Re}\left\{\sum_{\nu, \mu=1}^{n} \alpha_{\nu} \alpha_{\mu} \log \frac{F\left(\zeta_{\nu}\right)-F\left(\zeta_{\mu}\right)}{\zeta_{\nu}-\zeta_{\mu}}\right\} \geqq \sum_{\nu, \mu=1}^{n} \alpha_{\nu}^{*} \alpha_{\mu} \log \left(\frac{1-F^{*}\left(\zeta_{\nu}\right) F\left(\zeta_{\mu}\right)}{1-\zeta_{\nu}^{*} \zeta_{\mu}}\right),
$$

where the right-hand side is obviously real. Passing to the function inverse to $F(z)$, we have thus established the following result.

Let $f(z)$ be regular, univalent, and bounded by unity in $|z|<1$. If $z_{1}, \cdots, z_{n}$ are points in $|z|<1$ and $\alpha_{1}, \cdots, \alpha_{n}$ are complex constants such that $\alpha_{1}+\cdots$ $+\alpha_{n}=0$, then

$$
\left|\sum_{\nu, \mu=1}^{n} \alpha_{\nu} \alpha_{\mu} \log \frac{f\left(z_{\nu}\right)-f\left(z_{\mu}\right)}{z_{\nu}-z_{\mu}}\right| \leqq \sum_{\nu, \mu=1}^{n} \alpha_{\nu} \alpha_{\mu}^{*} \log \frac{1-f\left(z_{\nu}\right) f^{*}\left(z_{\mu}\right)}{1-z_{\nu} z_{\mu}{ }^{*}} .
$$

That the real part may be replaced by the absolute value is seen by multiplying all the $\alpha_{v}$ by a complex number of suitable argument.

If we set $\alpha_{1}=1, \alpha_{2}=-1, n=2$, and assume, in addition, $f(0)=0$, (6) yields

$$
\left|\log \frac{z^{2} f^{\prime}(z) f^{\prime}(0)}{f^{2}(z)}\right| \leqq \log \frac{1-|f(z)|^{2}}{1-|z|^{2}}
$$


and thus

$$
\frac{1-|z|^{2}}{1-|f(z)|^{2}} \leqq\left|\frac{z^{2} f^{\prime}(z) f^{\prime}(0)}{f^{2}(z)}\right| \leqq \frac{1-|f(z)|^{2}}{1-|z|^{2}} .
$$

3. From (6) it is possible to derive a set of necessary and sufficient conditions for the coefficients of a function which is univalent and bounded in the unit circle.

Let $f(z)=a_{0}+a_{1} z+a_{2} z^{2}+\cdots$ be regular in a neighborhood of $z=0$ and let $A_{n m}$ and $B_{n m}$ denote the polynomials in $a_{0}, a_{1}, \cdots$ given by the expansions

$$
\begin{aligned}
\log \frac{f(z)-f(\zeta)}{z-\zeta} & =\sum_{n, m=0}^{\infty} A_{n m} z^{n} \zeta^{m} & \left(A_{m n}=A_{n m}\right), \\
-\log \left(1-f^{*}(\zeta) f(z)\right) & =\sum_{n, m=0}^{\infty} B_{n m} z^{n} \zeta^{* m} & \left(B_{m n}=B_{n m}^{*}\right) .
\end{aligned}
$$

In order that $a_{0}, a_{1}, \cdots$ be the coefficients of a function which is univalent and bounded by unity in $|z|<1$ it is necessary and sufficient that the inequalities

$$
\left|\sum_{n, m=1}^{N} A_{n m} \sigma_{n} \sigma_{m}\right| \leqq \sum_{n=1}^{N} \frac{\left|\sigma_{n}\right|^{2}}{n}-\sum_{n, m=1}^{N} B_{n m} \sigma_{n} \sigma_{m}^{*}, \quad N=1,2, \cdots,
$$

be satisfied for any set of complex numbers $\sigma_{1}, \cdots, \sigma_{N}$.

To prove the necessity, we observe that, by (7) and (8),

$$
\begin{aligned}
\sum_{\nu, \mu=1}^{\lambda} \alpha_{\nu} \alpha_{\mu} \log \frac{f\left(z_{\nu}\right)-f\left(z_{\mu}\right)}{z_{\nu}-z_{\mu}} & =\sum_{\nu, \mu=1}^{\lambda} \alpha_{\nu} \alpha_{\mu}\left(\sum_{n, m=0}^{\infty} A_{n m} z_{\nu}^{n} z_{\mu}^{m}\right) \\
& =\sum_{n, m=0}^{\infty} A_{n m}\left(\sum_{\nu=1}^{\lambda} \alpha_{\nu} z_{\nu}\right)\left(\sum_{\nu=1}^{\lambda} \alpha_{\nu} z_{\nu}{ }^{n}\right), \\
\sum_{\nu, \mu=1}^{\lambda} \alpha_{\nu} \alpha_{\mu}^{*} \log \left(1-f^{*}\left(z_{\nu}\right) f\left(z_{\mu}\right)\right) & =-\sum_{\nu, \mu=1}^{\lambda} \alpha_{\nu} \alpha_{\mu}^{*}\left(\sum_{n, m=0}^{\infty} B_{n m} z_{\nu} z_{\mu}^{* m}\right) \\
& =-\sum_{n, m=0}^{\infty} B_{n m}\left(\sum_{\nu=1}^{\lambda} \alpha_{\nu} z_{\nu}^{n}\right)\left(\sum_{\nu=1}^{\lambda} \alpha_{\nu} z_{\nu}{ }^{*}\right)^{*} .
\end{aligned}
$$

Moreover,

$$
\sum_{\nu, \mu=1}^{\lambda} \alpha_{\nu} \alpha_{\mu}^{*} \log \left(1-z_{\nu} z_{\mu}^{*}\right)=-\sum_{n=1}^{\infty} \frac{1}{n}\left|\sum_{\nu=1}^{\lambda} \alpha_{\nu} z_{\nu}^{n}\right|^{2} .
$$

Hence, if we can show that it is possible to find complex numbers $\alpha_{1}, \alpha_{2}, \ldots$ and points $z_{1}, z_{2}, \cdots$ in $|z|<1$ such that, for given $\sigma_{1}, \cdots, \sigma_{N}$,

$$
\sum_{n=1}^{\infty} \alpha_{v} z_{p}{ }^{n}=\sigma_{n}, n=1, \cdots, N, \quad \sum_{n=1}^{\infty} \alpha_{p} z_{p}^{n}=0, \quad n=0, N+1, \cdots,
$$


(9) will follow from (6). Now the equations (10) are equivalent to the existence of an expansion

$$
\sum_{n=1}^{N} \sigma_{n} t^{n}=\sum_{\nu=1}^{\infty} \frac{\alpha_{\nu}}{1-z_{\nu} t}
$$

which converges in a neighborhood of $t=0$. To establish (11), we choose the $z_{\nu}$ such that $\lim _{\nu \rightarrow \infty} z_{\nu}=z_{0},\left|z_{0}\right|<1$, and we observe that the functions $\left(1-z_{\nu} t\right)^{-1}$ form a complete set in the space $L_{2}$ of functions which are regular in $|t|<1$ and satisfy $\int_{|t|=1}|f(t)|{ }^{2}|d t|<\infty$. Indeed, if there were a function of $L_{2}$, say $g(z)$, which is orthogonal to all functions of the set, we would have

$$
0=\int_{|t|=1} \frac{g(t)|d t|}{1-z_{\nu}^{*} t^{*}}=\frac{1}{i} \int_{|t|=1} \frac{g(t) d t}{t-z_{\nu}^{*}}=2 \pi g\left(z_{\nu}^{*}\right),
$$

and, since $\lim z_{\nu}{ }^{*}=z_{0}{ }^{*}$ and $g(t)$ is regular at $t=z_{0}{ }^{*}$, it would follow that $g(t)$ vanishes identically. From the completeness of the set $\left(1-z_{\nu} t\right)^{-1}$ follows the existence of a converging expansion of the type (11) for all functions of $L_{2}$ [1]. This proves (11) and thus also the necessity of the conditions (9).

To prove sufficiency, set $\sigma_{n}=z^{n}+\zeta^{n}, n=1, \cdots, N, \sigma_{n}=0, n>N$. For $N \rightarrow \infty$ it then follows from the positivity of the right-hand side of (9) that $\log \left[\left(1-|z|^{2}\right)\left(1-|\zeta|^{2}\right)\left|1-\zeta^{*} z\right|^{2}\right] \leqq \log \left[\left(1-|f(z)|^{2}\right)\left(1-|f(\zeta)|^{2}\right) \mid 1\right.$ $\left.-\left.f^{*}(\zeta) f(z)\right|^{2}\right]$. Letting $\zeta \rightarrow z$, we find that $|f(z)|<1$. The same set $\sigma_{n}$ will also assure the univalence of $f(z)$. Neglecting the nonpositive second term on the righthand side of (9), we obtain

$$
\begin{aligned}
\left|\log f^{\prime}(z)+\log f^{\prime}(\zeta)+2 \log \frac{f(z)-f(\zeta)}{z-\zeta}\right| & \\
\leqq & \log \left[\frac{1}{\left(1-|z|^{2}\right)\left(1-|\zeta|^{2}\right)\left|1-\zeta^{*} z\right|^{2}}\right],
\end{aligned}
$$

which shows that $f(z) \neq f(\zeta)$ if $z \neq \zeta$ and both are in the unit circle.

If we consider the class of univalent functions in the unit circle for which $|f(z)|<M$ and let $M \rightarrow \infty$, we obtain the corresponding results for univalent functions without any boundedness restrictions. It is easily confirmed that (6) is in this case replaced by

$$
\left|\sum_{\nu, \mu=1}^{n} \alpha_{\nu} \alpha_{\mu} \log \frac{f\left(z_{\nu}\right)-f\left(z_{\mu}\right)}{z_{\nu}-z_{\mu}}\right| \leqq \sum_{\nu, \mu=1}^{n} \alpha_{\nu} \alpha_{\mu}{ }^{*} \log \frac{1}{1-z_{\nu} z_{\mu}{ }^{*}}
$$

and (9) takes the form

$$
\left|\sum_{n, m=1}^{N} A_{n m} \sigma_{n} \sigma_{m}\right| \leqq \sum_{n=1}^{N} \frac{\left|\sigma_{n}\right|^{2}}{n},
$$

where $A_{n m}$ is given by (7). ( $\left.9^{\prime}\right)$ is equivalent to Grunsky's necessary and suffi- 
cient conditions [9] for the coefficients of a function univalent in the unit circle.

It is of interest to find the cases in which the sign of equality will occur in (6). Since (6) is but a special case of (1), this will happen if the function $p(z)$ constructed with the singularity (4) will be the same for both $D$ and $D_{1}$. If $D_{1}$ is the unit circle, the function $p_{1}(z)$ with the singularity (4) is of the form

$$
p_{1}(z)=\operatorname{Re}\left\{\sum_{\nu=1}^{n}\left[\alpha_{\nu} \log \left(z-\zeta_{\nu}\right)-\alpha_{\nu}^{*} \log \left(1-\zeta_{\nu}^{*} z\right)\right]\right\} .
$$

Hence, $p(z)$ will be identical with $p_{1}(z)$ if $D$ is a simply-connected domain whose boundary is either the circumference $|z|=1$ or consists of parts of $|z|=1$ and one or more arcs whose equation is

$$
\operatorname{Re}\left\{\sum_{\nu=1}^{n}\left[\alpha_{\nu} \log \left(z-\zeta_{\nu}\right)-\alpha_{\nu}^{*} \log \left(1-\zeta_{\nu}^{*} z\right)\right]\right\}=0 .
$$

It follows that there will be equality in (6) if $w=f(z)$ maps the unit circle onto a domain within $|w|<1$ whose boundary consists of parts either satisfying $|w|=1$ or

$$
\operatorname{Re}\left\{\sum_{\nu=1}^{n}\left[\beta \alpha_{\nu} \log \left(w-f\left(z_{\nu}\right)\right)-\beta^{*} \alpha_{\nu}^{*} \log \left(1-f^{*}\left(z_{\nu}\right) w\right)\right]\right\}=0,
$$

where $\beta$ is a complex number of suitable argument.

4. Another application of Theorem I concerns the Bergman kernel function $K(z, \zeta)$ of a plane domain $D[1]$. It was shown by Schiffer [15] that

$$
K(z, \zeta)=-(2 / \pi)\left(\partial^{2} g(z, \zeta) / \partial z \partial \zeta^{*}\right)
$$

where $g(z, \zeta)$ is the Green's function of $D$ and the differential operators $\partial / \partial z$ and $\partial / \partial z^{*}$ are defined by

$$
\frac{\partial}{\partial z}=\frac{1}{2}\left(\frac{\partial}{\partial x}-i \frac{\partial}{\partial y}\right), \quad \frac{\partial}{\partial z^{*}}=\frac{1}{2}\left(\frac{\partial}{\partial x}+i \frac{\partial}{\partial y}\right), \quad z=x+i y .
$$

Schiffer also showed that the function

$$
L(z, \zeta)=-\frac{2}{\pi} \frac{\partial^{2} g(z, \zeta)}{\partial z \partial \zeta}=\frac{1}{\pi(z-\zeta)^{2}}+l(z, \zeta)
$$

plays a fundamental role in the theory of conformal mapping. Both $K(z, \zeta)$ and the function $l(z, \zeta)$ defined in (13) are regular in $D$.

If $\alpha_{1}, \cdots, \alpha_{n}$ are complex parameters, then

$$
p(z)=\operatorname{Re}\left\{\sum_{\nu=1}^{n} \alpha_{\nu} \frac{\partial g\left(z, \zeta_{\nu}\right)}{\partial \zeta_{\nu}}\right\}
$$


vanishes on $C$ and may be identified with the function $p(z)$ of Theorem I. The corresponding singularity function is

$$
S(z)=\operatorname{Re}\left\{\sum_{\nu=1}^{n} \alpha_{\nu} \frac{\partial \log \left|z-\zeta_{\nu}\right|}{\partial \zeta_{\nu}}\right\}=-\frac{1}{2} \operatorname{Re}\left\{\sum_{\nu=1}^{n} \frac{\alpha_{\nu}}{z-\zeta_{\nu}}\right\} .
$$

If $q(z, \zeta)$ denotes the analytic function in $z$ for which $\operatorname{Re}\{q(z, \zeta)\}=g(z, \zeta)$, we obviously have

$$
p(z)=\frac{1}{2} \operatorname{Re}\left\{\sum_{\nu=1}^{n}\left[\alpha_{\nu} \frac{\partial q\left(z, \zeta_{\nu}\right)}{\partial \zeta_{\nu}}+\alpha_{\nu}^{*} \frac{\partial q\left(z, \zeta_{\nu}\right)}{\partial \zeta_{\nu}^{*}}\right]\right\},
$$

where the expression enclosed in brackets is single-valued in $D$, since the periods of $q(z, \zeta)$ are pure imaginary. Since $p(z)$ vanishes on $C$, we may thus evaluate the integral (1) by means of the residue theorem in the same way as in (5). We obtain

$$
\begin{aligned}
\int_{C} S(z) \frac{\partial p(z)}{\partial n} d s=-\operatorname{Re}\left\{\frac { 1 } { 2 i } \int _ { C } \left(\sum_{\nu=1}^{n} \alpha_{\nu}\left[\frac{\partial q\left(z, \zeta_{\nu}\right)}{\partial \zeta_{\nu}}-\frac{1}{z-\zeta_{\nu}}\right]\right.\right. \\
\left.\left.+\alpha_{\nu}^{*} \frac{\partial q\left(z, \zeta_{\nu}\right)}{\partial \zeta_{\nu}^{*}}\right)\left(\sum_{\mu=1}^{n} \frac{\alpha_{\mu}}{\left(z-\zeta_{\mu}\right)^{2}}\right) d z\right\} \\
=-\pi \operatorname{Re}\left\{\sum_{\nu, \mu=1}^{n} \alpha_{\nu} \alpha_{\mu}\left[\frac{\partial^{2} q\left(\zeta_{\mu}, \zeta_{\nu}\right)}{\partial \zeta_{\nu} \partial \zeta_{\mu}}+\frac{1}{\left(\zeta_{\mu}-\zeta_{\nu}\right)^{2}}\right]\right. \\
\left.+\sum_{\nu, \mu=1}^{n} \alpha_{\nu}^{*} \alpha_{\mu} \frac{\partial^{2} q\left(\zeta_{\mu}, \zeta_{\nu}\right)}{\partial \zeta_{\mu} \partial \zeta_{\nu}}\right\} .
\end{aligned}
$$

In view of (12), (13), and the fact that

$$
\frac{\partial q(z, \zeta)}{\partial z}=2 \frac{\partial g(z, \zeta)}{\partial z},
$$

it thus follows from Theorem I that the quantity

$$
\operatorname{Re}\left\{\sum_{\nu, \mu=1}^{n} \alpha_{\nu} \alpha_{\mu}^{*} K\left(\zeta_{\nu}, \zeta_{\mu}\right)-\sum_{\nu, \mu=1}^{n} \alpha_{\nu} \alpha_{\mu} l\left(\zeta_{\nu}, \zeta_{\mu}\right)\right\}
$$

decreases if $D$ increases, a result derived by Bergman and Schiffer [2] by means of a variational method.

3. Single-valued conjugates. 1 . In this section we derive a variant of Theorem I which is useful in the treatment of extremal problems involving single-valued analytic functions in multiply-connected domains or in domains not of genus zero.

TheOREM II. Let $R, D, D_{1}, C, C_{1}$, and $S(z)$ have the same meaning as in Theorem I. Let $p(z)$ denote the function which takes constant values on the indi- 
vidual components of $C$ and is such that $p(z)+S(z)$ is harmonic in $D$ and, moreover, satisfies

$$
\int_{\Gamma} \frac{\partial(p(z)+S(z))}{\partial n} d s=0
$$

for any closed contour $\Gamma$ in $D$. If $p_{1}(z)$ is the corresponding function associated with $D_{1}$, then

$$
\int_{C} S(z) \frac{\partial p(z)}{\partial n} d s \geqq \int_{C_{1}} S(z) \frac{\partial p_{1}(z)}{\partial n} d s .
$$

The proof of Theorem II is similar to that of Theorem I, the only difference being that the ordinary minimum property of the Dirichlet integral is replaced by the following more general inequality applicable to harmonic functions $u(z)$ which have a single-valued harmonic conjugate in $D$, that is, functions $u(z)$ such that

$$
\int_{\Gamma} \frac{\partial u(z)}{\partial n} d s=0
$$

for every closed contour $\Gamma$ in $D$.

Let $u(z)$ be harmonic in $D$ and satisfy (19) for every closed contour in $D$, and let $v(z)$ be continuous in $D$ with the possible exception of the points of a finite number of closed contours $A_{\mu}$ in $D$ at which $v(z)$ may have a constant jump across $A_{\mu}$; furthermore, let $v(z)$ have continuous first derivatives in $D$ with the possible exception of the points of a finite number of closed contours in $D$. If, on the individual components $C_{\nu}$ of the boundary $C$ of $D, u(z)-v(z)=$ const., then

$$
(u, u)_{D} \leqq(v, v)_{D}
$$

with equality only for $u(z)=v(z)$.

Indeed, we have

$$
\begin{aligned}
(u, u-v)_{D} & =\sum_{\nu} \int_{C_{\nu}}(u-v) \frac{\partial u}{\partial n} d s+\sum_{\mu} \int_{A_{\mu}}(u-v) \frac{\partial u}{\partial n} d s \\
& =\sum_{\nu} \text { const. } \int_{C_{\nu}} \frac{\partial u}{\partial n} d s+\sum_{\mu} \text { const. } \int_{A_{\mu}} \frac{\partial u}{\partial n} d s,
\end{aligned}
$$

and this vanishes by (19). Hence,

$$
\begin{aligned}
(v, v)_{D} & =(u, u)_{D}+2(u, u-v)_{D}+(u-v, u-v)_{D} \\
& =(u, u)_{D}+(u-v, u-v)_{D} \geqq(u, u)_{D},
\end{aligned}
$$

which proves (20).

To prove Theorem II, we set $u(z)=S(z)+p_{1}(z)$ in $D_{1}$ and define $v(z)$ by 


$$
v(z)=S(z)+p(z), \quad z \in D ; \quad v(z)=S(z), \quad z \in D_{1}-D .
$$

This choice obviously satisfies the conditions under which (20) holds. Hence,

$$
(S+p, S+p)_{D}+(S, S)_{D_{1}-D} \geqq\left(S+p_{1}, S+p_{1}\right)_{D_{1}}
$$

and thus, by Green's formula,

$$
\begin{aligned}
\int_{C} S \frac{\partial S}{\partial n} d s+\int_{C} S \frac{\partial p}{\partial n} d s & +\int_{C} p \frac{\partial(S+p)}{\partial n} d s+\int_{C_{1}} S \frac{\partial S}{\partial n} d s-\int_{C} S \frac{\partial S}{\partial n} d s \\
& \geqq \int_{C_{1}} S \frac{\partial S}{\partial n} d s+\int_{C_{1}} S \frac{\partial p_{1}}{\partial n} d s+\int_{C_{1}} p_{1} \frac{\partial(S+p)}{\partial n} d s .
\end{aligned}
$$

Observing (17) and the fact that $p(z)$ and $p_{1}(z)$ take constant values on the components of $C$ and $C_{1}$, respectively, we arrive at (18).

2. To illustrate the application of Theorem II, consider the case of a finite multiply-connected plane domain $D$ and of the analytic function $F(z)=F(z, \zeta)$ which maps $D$ onto the schlicht unit circle with concentric circular slits. If $\zeta$ is a point of $D, F(z, \zeta)$ may be so normalized as to satisfy $F(\zeta, \zeta)=0$ and we may require that the outer boundary of $D$ be transformed into the unit circumference. The argument of $(z-\zeta)^{-1} F(z, \zeta)$ returns to its initial value if $z$ describes a boundary component $C_{\nu}$ of $D$, and $|F(z, \zeta)|$ is constant on each $C_{\boldsymbol{r}}$. We may therefore set $-\log |z-\zeta|=S(z)$ and $\log |F(z, \zeta)|=p(z)$ and apply Theorem II. It follows that

$$
-\int_{c} \log |z-\zeta| \frac{\partial}{\partial n} \log |F(z, \zeta)| d s
$$

decreases if $D$ increases. To evaluate (21), we observe that

$$
\int_{C_{\boldsymbol{\nu}}} \frac{\partial}{\partial n} \log |F(z, \zeta)| d s=\int_{C_{\boldsymbol{\nu}}} d\{\arg F(z, \zeta)\}=0
$$

if $C_{\nu}$ is an inner boundary component and that $\log |F(z, \zeta)|=0$ on the outer boundary. (21) may therefore be replaced by

$$
\begin{aligned}
-\int_{C}[\log |z-\zeta| & -\log |F(z, \zeta)|] \frac{\partial}{\partial n} \log |F(z, \zeta)| d s \\
& =\operatorname{Re}\left\{\frac{1}{i} \int_{C}\left(\log \frac{F(z, \zeta)}{z-\zeta}\right) \frac{F^{\prime}(z, \zeta)}{F(z, \zeta)} d z\right\}=2 \pi \log \left|F^{\prime}(\zeta, \zeta)\right| .
\end{aligned}
$$

Hence, $\left|F^{\prime}(\zeta, \zeta)\right|$ decreases if $D$ increases.

If $D_{1}$ is the unit circle and $\zeta$ its center, then $F_{1}(z, \zeta) \equiv z$. It thus follows from the monotonicity of $\left|F^{\prime}(\zeta, \zeta)\right|$ that $\left|F^{\prime}(z, 0)\right| \geqq 1$ if $D$ is a schlicht multiply-connected domain contained in the unit circle. This is equivalent to the following well known theorem on bounded univalent functions $[6 ; 8]$ : Let 
$G$ be a finite plane domain and let $f(z)$ be univalent and $|f(z)| \leqq 1$ in G. If, moreover, $f(z)$ maps the outer boundary of $G$ onto the outer boundary of the image domain, then

$$
\left|f^{\prime}(\zeta)\right| \leqq\left|F^{\prime}(\zeta, \zeta)\right|
$$

where $F(z, \zeta)$ maps $G$ onto the unit circle with concentric circular slits.

3. As another illustration, we consider the analytic function $F_{\nu \mu}(z)$ which maps a schlicht domain of connectivity $\geqq 3$ onto the circular ring $1<|w|<M$ minus a number of concentric circular slits, where the subscripts $\nu, \mu$ indicate that the boundary components $C_{\nu}$ and $C_{\mu}$ are transformed into the circumferences $|w|=1$ and $|w|=M$, respectively. We denote by $D_{1}$ the domain containing $D$ which is bounded by $C_{\nu}$ and $C_{\mu}$, and by $F_{0}(z)$ the function mapping $D_{1}$ onto the circular ring $1<|w|<M_{0}$. Clearly, $\log F_{\nu \mu}(z)-\log F_{0}(z)$ is regular and single-valued in $D$ and we may apply Theorem II with $S(z)$ $=-\log \left|F_{0}(z)\right|, p(z)=\log \left|F_{\nu \mu}(z)\right|, p_{1}(z)=\log \left|F_{0}(z)\right|$. By (18), we have

$$
\int_{C} \log \left|F_{0}\right| \frac{\partial}{\partial n} \log \left|F_{\nu \mu}\right| d s \leqq \int_{C_{\nu}+C_{\mu}} \log \left|F_{0}\right| \frac{\partial}{\partial n} \log \left|F_{0}\right| d s .
$$

Since both $\left|F_{0}\right|$ and $\left|F_{\nu \mu}\right|$ are harmonic in $D$, the left-hand side of (22) is, by Green's formula, equal to

$$
\int_{C} \log \left|F_{\nu \mu}\right| \frac{\partial}{\partial n} \log \left|F_{0}\right| d s .
$$

$\left|F_{\nu \mu}\right|$ is constant on each boundary component and

$$
\int \frac{\partial}{\partial n} \log \left|F_{0}\right| d s=0
$$

on all boundary components other than $C_{\nu}$ and $C_{\mu}$. Since $\left|F_{\nu \mu}\right|=1$ on $C_{\nu}$, $\left|F_{\nu \mu}\right|=M$ on $C_{\mu},\left|F_{0}\right|=1$ on $C_{\nu}$, and $\left|F_{0}\right|=M_{0}$ on $C_{\mu}$, (22) thus reduces to

$$
\log M \int_{C_{\mu}} \frac{\partial}{\partial n} \log \left|F_{0}\right| d s \leqq \log M_{0} \int_{C_{\mu}} \frac{\partial}{\partial n} \log \left|F_{0}\right| d s .
$$

In view of

$$
\begin{aligned}
\int_{C_{\mu}} \frac{\partial}{\partial n} \log \left|F_{0}\right| & =\frac{1}{\log M_{0}} \int_{C_{\nu}+C_{\mu}} \log \left|F_{0}\right| \frac{\partial}{\partial n} \log \left|F_{0}\right| d s \\
& =\left(\log \left|F_{0}\right|, \log \left|F_{0}\right|\right)_{D_{0}}>0,
\end{aligned}
$$

this shows that $M<M_{0}$. This is equivalent to the following result $[4 ; 6]$ : Let $D$ be a schlicht domain of connectivity $\geqq 3$ and let $C_{\nu}$ and $C_{\mu}$ denote two of its boundary components. If $M$ denotes the Riemann modulus of the doubly- 
connected domain $D_{0}$ bounded by $C_{\nu}$ and $C_{\mu}$, then, within the conformal class of $D$, the problem $M=\max$ is solved by the domain whose boundary components other than $C_{\nu}$ and $C_{\mu}$ are transformed into concentric circular slits about the origin by the conformal mapping carrying $D_{0}$ into a circular ring about the origin.

4. Positive functionals. 1. The monotonicity properties indicated in Theorems I and II were derived without any assumptions regarding the behavior of the singularity function $S(z)$ outside the larger of the two domains involved. The following theorem indicates an inequality which holds if an additional assumption of this type is made.

ThEOREM III. Let $R$ be a closed Riemann surface of finite genus and let $D$ be a domain embedded in $R$ and bounded by a finite number of closed analytic curves, $C_{1}, \cdots, C_{n}\left(C_{1}+\cdots+C_{n}=C\right)$, all of which can be contracted to points within $R-D$. Let further $S(z)$ denote a real Abelian integral on $R$ all of whose singularities are situated in $D$, and which is single-valued in each component of $R-D$. If $p(z)$ is a real function for which $p(z)-S(z)$ is singlevalued and harmonic in $D$ and which takes constant boundary values on each $C_{\nu}$, then

$$
\int_{C}[p(z)-S(z)] \frac{\partial p(z)}{\partial n} d s \geqq 0 .
$$

Equality in (23) will hold if, and only if, $p(z)=S(z)$, in which case $D$ is bounded by analytic slits given by $S(z)=$ const.

The proof of Theorem III is very simple. Since $p-S$ is harmonic and single-valued in $D$, the Dirichlet integral of this function can be transformed by means of Green's formula. We have

$$
\begin{aligned}
&(p-S,p-S)_{D}=\int_{C}(p-S) \frac{\partial}{\partial n}(p-S) d s \\
& \quad=\int_{C}(p-S) \frac{\partial p}{\partial n} d s-\sum_{\nu=1}^{n} p \int_{C_{\nu}} \frac{\partial S}{\partial n} d s+\int_{C} S \frac{\partial S}{\partial n} d s,
\end{aligned}
$$

where the fact that $p=$ const. on $C_{\boldsymbol{v}}$ has been used. If $D^{\prime}$ denotes the complement of $D$ with respect to $R, S$ is single-valued and harmonic in $D^{\prime}$ and we have, by Green's formula,

$$
\int_{C} S \frac{\partial S}{\partial n} d s=-(S, S)_{D^{\prime}}
$$

The integrals over the $C_{\nu}$ vanish since the $C_{\nu}$ can be contracted to points in $D^{\prime}$ and $S$ is harmonic and single-valued there. Hence,

$$
(p-S, p-S)_{D}+(S, S)_{D^{\prime}}=\int_{C}(p-S) \frac{\partial p}{\partial n} d s .
$$


Since the Dirichlet integrals are non-negative, this proves (23). To have equality in (23), both Dirichlet integrals in (25) must vanish, which obviously is possible only if $p=S$ and if $D^{\prime}$ has the area 0 , that is, if $D$ is a slit domain. Since $p=S$ and $p$ is constant on each $C$, the boundary slits will then have the equations $S(z)=$ const. This proves Theorem III.

2. Before we apply Theorem III to a number of problems in the theory of functions, we indicate a somewhat stronger form of this result which holds if the function $p(z)$ has the value zero on the entire boundary of $D$.

Theorem IIIa. If the function $p(z)$ takes the boundary values zero on $C$, the conclusion of Theorem III will hold regardless of the homotopic type of the closed curves $C_{\nu}$.

Indeed, the assumption that the $C_{\nu}$ can be contracted to points within $D^{\prime}$ was used only to guarantee the vanishing of the integrals over the $C_{v}$ in (24). Since now $p(z)=0$ for $z \in C_{\nu}$, these integrals have zero factors and the argument goes through as before.

As an application of Theorem IIIa, consider the case in which $S(z)$ is the single-valued real Abelian integral of the third kind $t(z ; \zeta, \eta)$ which has the local expansions $t(z ; \zeta, \eta)=\log |z-\zeta|+\cdots$ and $t(z ; \zeta, \eta)=-\log |z-\eta|$ $+\cdots$, respectively, near its logarithmic poles at $\zeta$ and $\eta$. If $\zeta$ and $\eta$ are in $D, p(z)$ will be of the form $p(z)=g(z, \eta)-g(z, \zeta)$, where $g$ denotes the Green's function of $D$. By (23), we have

$$
\int_{C}[g(z, \eta)-g(z, \zeta)-t(z ; \zeta, \eta)] \frac{\partial}{\partial n}[g(z, \eta)-g(z, \zeta)] d s \geqq 0 .
$$

If we write

$$
g(z, \zeta)-g(z, \eta)+t(z ; \zeta, \eta)=H(z ; \zeta, \eta),
$$

$H(z ; \zeta, \eta)$ is harmonic and single-valued in $D$ and it follows from (26) and Green's formula that

$$
H(\eta ; \zeta, \eta)-H(\zeta ; \zeta, \eta) \geqq 0 .
$$

Since $g(z, \zeta)=g(\zeta, z)$ and $t(z ; \eta, \zeta)=-t(z ; \zeta, \eta)$, it follows from the definition (27) that this may also be written in the form

$$
H(\eta ; \eta, \zeta)+H(\zeta ; \zeta, \eta) \geqq 0 .
$$

We thus have the following result.

Let $D$ be a domain embedded in a closed Riemann surface of finite genus and let $t(z ; \zeta, \eta)$ denote the normalized single-valued real Abelian integral of the third kind on $R$ whose positive and negative logarithmic poles are at the points $\zeta$ and $\eta$ of $D$, respectively. If $g(z, \zeta)$ denotes the Green's function of $D$ and $H(z ; \zeta, \eta)$ is defined by (27), the inequality (28) holds. Equality in (28) will occur if, and only if, the boundary of $D$ consists of slits along arcs satisfying $t(z ; \zeta, \eta)=0$. 
If $R$ is the schlicht plane, $t(z ; \zeta, \eta)$ reduces to $\log |z-\zeta|-\log |z-\eta|$. With $h(z, \zeta)=g(z, \zeta)+\log |z-\zeta|,(28)$ is in this case equivalent to

$$
h(\zeta, \zeta)+h(\eta, \eta) \leqq 2 h(\zeta, \eta)
$$

derived by Schiffer [14] by means of Hadamard's variation formula for the Green's function. Except for the trivial case $\zeta=\eta$, there will be equality in $\left(28^{\prime}\right)$ if the schlicht domain $D$ is bounded by one or more rectilinear slits situated along the line of symmetry $|z-\zeta|=|z-\eta|$ of $\zeta$ and $\eta$.

3. Passing now to applications of Theorem III, we first remark that in the case in which the function $p(z)-S(z)$ has a single-valued conjugate in $D$ the left-hand side of (23) can be expressed by means of a Cauchy integral. Since $p=$ const. on each $C_{\nu}, \partial p / \partial n$ is pure imaginary on $C$. Writing $p(z)$ $=\operatorname{Re}\{q(z)\}, S(z)=\operatorname{Re}\{\sigma(z)\}$, we thus have

$$
\partial p / \partial n=(1 / i) q^{\prime}(z) d z / d s,
$$

$z \in C$,

and (23) takes the form

$$
\operatorname{Re}\left\{\frac{1}{i} \int_{C}[q(z)-\sigma(z)] q^{\prime}(z) d z\right\} \geqq 0 .
$$

If $q-\sigma$ is single-valued, this integral can be evaluated by means of the residue theorem, which also shows that (29) may be replaced by

$$
\operatorname{Re}\left\{\frac{1}{i} \int_{C}[q(z)-\sigma(z)] \sigma^{\prime}(z) d z\right\} \geqq 0 .
$$

Let now $R$ be the schlicht plane and let $D$ contain the point at infinity. By $w=f(z)$ we denote the univalent function in $D$ which, near $z=\infty$, is normalized by $f(z)=z+a_{0}+a_{1} z^{-1}+\cdots$ and which maps $D$ onto a domain bounded by slits along arcs of the equation

$$
\operatorname{Re}\left\{\sum_{\mu=1}^{m} \alpha_{\mu} \log \left(w-w_{\mu}\right)\right\}=\text { const., }
$$

where the $\alpha_{\mu}$ and $w_{\mu}$ are arbitrary complex constants. If the points $w_{\mu}$ do not lie on any of the slits, there are points $\zeta_{\nu}$ in $D$ such that $f\left(\zeta_{\mu}\right)=w_{\mu}$. The functions

$$
\sigma(z)=\sum_{\mu=1}^{m} \alpha_{\mu} \log \left(z-\zeta_{\mu}\right), \quad q(z)=\sum_{\mu=1}^{m} \alpha_{\mu} \log \left[f(z)-f\left(\zeta_{\mu}\right)\right]
$$

may be used in (30). Indeed, $\operatorname{Re}\{\sigma(z)\}=$ const. on each boundary component and

$$
\sum_{\mu=1}^{m} \alpha_{\mu} \log \frac{f(z)-f\left(\zeta_{\mu}\right)}{z-\zeta_{\mu}}
$$


is regular and, since the boundary curves of $D$ and their conformal images do not surround the points $\zeta_{\mu}$ and $f\left(\zeta_{\mu}\right)$, respectively, single-valued in $D$. The inequality (30) takes the form

$$
\operatorname{Re}\left\{\frac{1}{i} \int_{C}\left[\sum_{\mu=1}^{m} \alpha_{\mu} \log \frac{f(z)-f\left(\zeta_{\mu}\right)}{z-\zeta_{\mu}}\right]\left[\sum_{\mu=1}^{m} \frac{\alpha_{\mu}}{z-\zeta_{\mu}}\right] d z\right\} \geqq 0 .
$$

Near $z=\infty$, the residue of the integrand is zero since $f(z) / z \rightarrow 1$ if $z \rightarrow \infty$. Hence, the residue theorem yields the inequality

$$
\operatorname{Re}\left\{\sum_{\nu, \mu=1}^{m} \alpha_{\nu} \alpha_{\mu} \log \frac{f\left(\zeta_{\nu}\right)-f\left(\zeta_{\mu}\right)}{\zeta_{\nu}-\zeta_{\mu}}\right\} \geqq 0 .
$$

Let now $D^{\prime}$ be a domain of the same conformal class as $D$ which contains the point at infinity and is mapped onto $D$ by a function $z=g(u)$ with the normalization $g(u)=u+b_{0}+b_{1} u^{-1}+\ldots$. If we write $f[g(u)]=F(u)$, $g\left(u_{\nu}\right)=\zeta_{\nu},(32)$ is seen to be equivalent to

$$
\operatorname{Re}\left\{\sum_{\nu, \mu=1}^{m} \alpha_{\nu} \alpha_{\mu} \log \frac{g\left(u_{\nu}\right)-g\left(u_{\mu}\right)}{u_{\nu}-u_{\mu}}\right\} \leqq \operatorname{Re}\left\{\sum_{\nu, \mu=1}^{m} \alpha_{\nu} \alpha_{\mu} \log \frac{F\left(u_{\nu}\right)-F\left(u_{\mu}\right)}{u_{\nu}-u_{\mu}}\right\} .
$$

We have thus proved the following result.

Let $\alpha_{1}, \cdots, \alpha_{m}$ be arbitrary complex constants and let $u_{1}, \cdots, u_{m}$ be points of a schlicht multiply-connected domain containing the point at infinity. The extremal problem

$$
\operatorname{Re}\left\{\sum_{\nu, \mu=1}^{m} \alpha_{\nu} \alpha_{\mu} \log \frac{g\left(u_{\nu}\right)-g\left(u_{\mu}\right)}{u_{\nu}-u_{\mu}}\right\}=\max
$$

within the family of univalent functions $g(u)$ in $D^{\prime}$ normalized by $g(u)=u+b_{0}$ $+b_{1} u^{-1}+\cdots$ is solved by a function $F(u)$ mapping $D^{\prime}$ onto a slit domain whose boundary slits satisfy

$$
\operatorname{Re}\left\{\sum_{\nu=1}^{m} \alpha_{\nu} \log \left[F(u)-F\left(u_{\nu}\right)\right]\right\}=\text { const., }
$$

provided such a mapping exists.

The question of the existence of the extremal map requires a special discussion which goes beyond the elementary methods used in the present paper. It can, however, be avoided by phrasing the result as follows:

Let $D$ be a schlicht domain containing the point at infinity which is bounded by slits satisfying (31). If $f(w)=w+c_{0}+O\left(w^{-1}\right)$ is univalent in $D$, then

$$
\operatorname{Re}\left\{\sum_{\nu, \mu=1}^{m} \alpha_{\nu} \alpha_{\mu} \log \frac{f\left(w_{\nu}\right)-f\left(w_{\mu}\right)}{w_{\nu}-w_{\mu}}\right\} \leqq 0,
$$

with equality only for $f(w)=w$. 
For $m=1, w_{1}=0,(31)$ reduces to $\operatorname{Re}\{\alpha \log w\}=$ const. and (33) to $\operatorname{Re}\left\{\alpha^{2} \log f^{\prime}(0)\right\} \leqq 0$, expressing the well known extremal properties of the spiral slit maps $[7 ; 8]$. Another geometrically simple extremal map is obtained by setting, in (33), $\alpha_{1}=\cdots=\alpha_{m}=e^{i \theta}$. We find that the problem

$$
\operatorname{Re}\left\{e^{2 i \theta} \log \prod_{\nu, \mu=1}^{m}\left(\frac{g\left(u_{\nu}\right)-g\left(u_{\mu}\right)}{u_{\nu}-u_{\mu}}\right)\right\}=\max
$$

is solved by a map whose boundary slits are arcs of trajectories intersecting the family of lemniscates with the foci $F\left(u_{1}\right), \cdots, F\left(u_{m}\right)$ at the angle $-\theta$.

4. Passing to another application of Theorem III, we consider the case of a schlicht domain $D$ containing the origin. We denote by $f(z)$ a function which is univalent in $D$, vanishes at the origin, and maps $D$ onto a domain bounded by slits along arcs satisfying $\operatorname{Re}\left\{P\left(w^{-1}\right)\right\}=$ const., where $P(t)$ is a given polynomial of degree $n$. If $Q(t)$ is another polynomial of the same degree such that

$$
P\left[\frac{1}{f(z)}\right]-Q\left[\frac{1}{z}\right]
$$

is regular at $z=0$, we may clearly set

$$
q(z)=P\left[\frac{1}{f(z)}\right], \quad \sigma(z)=Q\left[\frac{1}{z}\right]
$$

and apply (30). We obtain

$$
\operatorname{Re}\left\{\frac{1}{i} \int_{C}\left(P\left[\frac{1}{f(z)}\right]-Q\left[\frac{1}{z}\right]\right)\left(Q\left[\frac{1}{z}\right]\right)^{\prime} d z\right\} \geqq 0 .
$$

Let now $D^{\prime}$ be a schlicht domain containing the origin which is mapped onto $D$ by a function $z=g(u)$ for which $g(u)=0$. If $C^{\prime}$ denotes a circle about the origin which together with its interior is within $D^{\prime}$ and if we write

$$
f[g(u)]=F(u),
$$

(35) is equivalent to

$$
\operatorname{Re}\left\{\frac{1}{i} \int_{C^{\prime}}\left(P\left[\frac{1}{F(u)}\right]-Q\left[\frac{1}{g(u)}\right]\right)\left(Q\left[\frac{1}{g(u)}\right]\right)^{\prime} d u\right\} \geqq 0 .
$$

Expanding into power series, we have

$$
P\left[\frac{1}{F(u)}\right]=\sum_{\nu=1}^{n} \frac{A_{\nu}}{u^{\nu}}+\sum_{\mu=0}^{\infty} C_{\mu} u^{\mu}, \quad Q\left[\frac{1}{g(u)}\right]=\sum_{\nu=1}^{n} \frac{A_{\nu}}{u^{\nu}}+\sum_{\mu=0}^{\infty} B_{\mu} u^{\mu}
$$

where, in view of our assumptions, the coefficients of the negative powers 
must be the same in both cases. Inserting these expansions in (36) and using the residue theorem, we obtain

$$
\operatorname{Re}\left\{\sum_{\nu=1}^{n} \nu\left(B_{\nu}-C_{\nu}\right) A_{\nu}\right\} \geqq 0 .
$$

We have thus derived the following result concerning the coefficients of univalent functions.

Let $D$ be a schlicht domain containing the origin and let $F(z)=a_{1} z$ $+a_{2} z^{2}+\cdots$ be a univalent function mapping $D$ onto a domain bounded by slits satisfying $\operatorname{Re}\left\{P\left(w^{1}\right)\right\}=$ const., where $P(t)$ is a polynomial of degree $n$. Let further $A_{\nu}$ and $C_{\mu}$ be the coefficients determined by the local expansion

$$
P\left[\frac{1}{F(z)}\right]=\sum_{\nu=1}^{n} \frac{A_{\nu}}{z^{\nu}}+\sum_{\mu=0}^{\infty} C_{\mu} z^{\mu}
$$

If $f(z)$ is also univalent in $D$ and $g(0)=0$, and the polynomial $Q(t)$ is so chosen that the local expansion

$$
Q\left[\frac{1}{f(z)}\right]=\sum_{\nu=1}^{n} \frac{A_{\nu}}{z^{\nu}}+\sum_{\mu=0}^{\infty} B_{\mu} z^{\mu}
$$

agrees with (37) in the coefficients of the negative powers, then

$$
\operatorname{Re}\left\{\sum_{\nu=1}^{n} \nu B_{\nu} A_{\nu}\right\} \geqq \operatorname{Re}\left\{\sum_{\nu=1}^{n} \nu C_{\nu} A_{\nu}\right\} .
$$

Equality in (38) will occur if, and only if, $f(z)$ maps $D$ onto a domain bounded by slits satisfying $\operatorname{Re}\left\{Q\left(w^{-1}\right)\right\}=$ const.

If $D$ is the unit circle, the coefficients $C_{\nu}$ are easily determined. Since we only used the fact that the right-hand side of (37) has a constant real part on the boundary of $D$, we may in this case take it to be of the form

$$
\sum_{\nu=1}^{n} \frac{A_{\nu}}{z^{\nu}}-\sum_{\nu=1}^{n} A_{\nu}^{*} z^{\nu}
$$

regardless of whether this expression can be written in the form $P\left[\{F(z)\}^{-1}\right]$, where $P(t)$ is a polynomial and $F(z)$ is univalent. Hence $C_{\nu}=-A_{\nu}, \nu=1, \cdots$, $n$, and (38) becomes

$$
\operatorname{Re}\left\{\sum_{\nu=1}^{n} A_{\nu} B_{\nu}\right\} \leqq \sum_{\nu=1}^{n} \nu\left|A_{\nu}\right|^{2}
$$

or, if we replace $f(z)$ by $f\left(e^{i \theta} z\right)$ with suitable $\theta$,

$$
\left|\sum_{\nu=1}^{n} A_{\nu} B_{\nu}\right| \leqq \sum_{\nu=1}^{n} \nu\left|A_{\nu}\right|^{2}
$$


5. As an application of (39) we derive a result recently obtained by Springer [17] by means of a variational method. If

$$
z=g(w)=w+\frac{b_{1}}{w}+\frac{b_{2}}{w^{2}}+\frac{b_{3}}{w^{3}}+\cdots
$$

maps a schlicht domain containing $w=\infty$ onto $|z|>1$, then $\left|b_{3}\right| \leqq 1$, with equality only for a rectilinear slit domain. If

$$
w=h(z)=z+\frac{a_{1}}{z}+\frac{a_{2}}{z^{2}}+\frac{a_{3}}{z^{3}}+\cdots
$$

is inverse to (40), it is easily found that

$$
-b_{3}=a_{3}+a_{1}^{2} \text {. }
$$

On the other hand

$$
h^{2}(z)=z^{2}+2 a_{1}+2 a_{2} z^{-1}+\left(2 a_{3}+\stackrel{2}{a}\right)_{1} z^{-2}+\cdots
$$

and thus, by (39),

$$
\left|2 a_{3}+a_{1}^{2}\right| \leqq 1
$$

Hence, by (41),

$$
2\left|b_{3}\right| \leqq 1+\left|a_{1}\right|^{2} \text {. }
$$

By a classical result - and also as an immediate consequence of (39)- $\left|a_{1}\right|<1$ unless $D$ is bounded by a rectilinear slit. It follows that $\left|b_{3}\right|<1$ except in this case.

6. As another application consider a function

$$
q(z)=z+\frac{c_{1}}{z}+\frac{c_{3}}{z^{3}}+\frac{c_{5}}{z^{5}}+\cdots,
$$

which is univalent in $|z|>1$. We have

$$
q^{3}(z)-3 c_{1} q(z)=z^{3}+3 c_{3} z^{-1}+\left(3 c_{5}+3 c_{1} c_{3}+c_{1}^{3}\right) z^{-3}+\cdots
$$

and therefore, by (39),

$$
\left|3 c_{5}+3 c_{1} c_{3}+c_{1}^{3}\right| \leqq 1
$$

If

$$
p(z)=z+a_{0}+\frac{a_{1}}{z}+\frac{a_{2}}{z^{2}}+\cdots
$$

is univalent and $f(z) \neq 0$ in $|z|>1$, the function 


$$
\left(f\left(z^{2}\right)\right)^{1 / 2}=z+\frac{a_{0}}{2} z^{-1}+\left(\frac{a_{1}}{2}-\frac{a_{0}}{8}\right) z^{-3}+\left(\frac{a_{2}}{2}-\frac{a_{0} a_{1}}{4}+\frac{a_{0}^{3}}{16}\right) z^{-5}+\cdots
$$

is also univalent in $|z|>1$ and we may apply (42). This yields

$$
\left|\frac{3}{2} a_{2}+\frac{a_{0}^{3}}{8}\right| \leqq 1 \text {. }
$$

Let now

$$
f(z)=z+\frac{a_{1}}{z}+\frac{a_{2}}{z^{2}}+\cdots
$$

be univalent in $|z|>1$. If $f(z) \neq \alpha$ in $|z|>1$, we may identify $f(z)-\alpha$ with the function $p(z)$ in (43). Hence, by (44),

$$
\left|\frac{3}{2} a_{2}-\frac{\alpha^{3}}{8}\right| \leqq 1 \text {, }
$$

if $\alpha$ is any value not taken by $f(z)$ in $|z|>1$. Replacing, if necessary, $f(z)$ by $e^{-i \gamma} f\left(e^{i \gamma} z\right)$, we may assume that $a_{2} \geqq 0$. If there exists a value $\alpha$, not taken by $f(z)$ in $|z|>1$, such that $\operatorname{Re}\left\{\alpha^{3}\right\}<0$, it will follow from (46) that $\left|a_{2}\right|<2 / 3$. Suppose, then, that for all such $\alpha$ we have $\operatorname{Re}\left\{\alpha^{3}\right\} \geqq 0$. If $\theta=\arg \alpha$, it follows that $\alpha$ is in one or more of the three angular regions $|\theta|<\pi / 6,|\theta-\pi / 3|$ $<\pi / 6,|\theta+\pi / 3|<\pi / 6$. If one of these regions did not contain values $\alpha$, all the values left out by $f(z)$ would be contained in a half-plane bounded by a line passing through the origin. This is impossible since, by (45), the center of gravity of the boundary of the image of $|z|>1$ is at the origin. Hence, all three angular spaces must contain boundary points of the image, which shows that the origin is also a boundary point. We may therefore take $\alpha=0$ in (46) and obtain $\left|a_{2}\right| \leqq 2 / 3$. This inequality, due to Schiffer [12] and Golusin [5], has thus been proved in all cases. The sign of equality will hold if, and only if, there is equality in the corresponding inequality (39). It is easily confirmed that, except for trivial transformations, this will occur only for the function $f(z)=z\left(1-z^{3}\right)^{-2 / 3}=z+2 z^{-2} / 3+\cdots$.

We add a remark concerning the coefficients of univalent functions $\phi(z)=z+\alpha_{2} z^{2}+\alpha_{3} z^{3}+\cdots$ which are regular in the unit circle. It is easily confirmed that (44) is equivalent to

$$
\left|\alpha_{4}-2 \alpha_{2} \alpha_{3}-\frac{13}{12} \alpha_{2}^{3}\right| \leqq \frac{2}{3}
$$

whence

$$
\left|\alpha_{4}\right| \leqq \frac{2}{3}+\left|2 \alpha_{2} \alpha_{3}-\frac{13}{12} \alpha_{2}^{3}\right|
$$


If it were true that the right-hand side of this inequality is maximized by the function for which $\alpha_{2}=2, \alpha_{3}=3$, it would follow that $\left|\alpha_{4}\right| \leqq 4$, in accordance with a well known conjecture. However, using the tables for the boundary surface of the $\left(\alpha_{2}, \alpha_{3}\right)$-space computed by Schaeffer and Spencer [11], it can be shown that the right-hand side is capable of values as large as $4.27\left({ }^{2}\right)$.

7. As a last application of (38) we derive the following result.

If

$$
f(z)=z+\frac{a_{1}}{z}+\frac{a_{2}}{z^{2}}+\frac{a_{3}}{z^{3}}+\cdots
$$

is the local expansion near $z=\infty$ of a function $f(z)$ which is univalent in a schlicht domain $D$ containing $z=\infty$, then the domain of variability of the expression $2 a_{3}+a_{1}^{2}$ for all such functions is a circle. To the points on the circumference correspond functions which map $D$ onto the full plane minus slits along arcs of $a$ number of concentric and coaxial equilateral hyperbolas.

If

$$
F_{\theta}(z)=z+\frac{b_{1}^{(\theta)}}{z}+\frac{b_{2}^{(\theta)}}{z^{2}}+\cdots
$$

yields such a hyperbolic slit mapping, where $\theta$ indicates that the axis of the hyperbolas forms the angle $\theta$ with the real axis, then $\operatorname{Re}\left\{\left[i e^{-2 i \theta} F_{\theta}(z)\right]^{2}\right\}$ $=$ const. on the boundary of $D$. Since

$$
f^{2}(z)=z^{2}+2 a_{1}+\frac{2 a_{2}}{z}+\frac{2 a_{3}+a_{1}^{2}}{z^{2}}+\cdots,
$$

it follows from (38) that

$$
\operatorname{Re}\left\{e^{-4 i \theta}\left(2 a_{3}+a_{1}^{2}\right)\right\} \leqq \operatorname{Re}\left\{e^{-4 i \theta}\left(2 b_{3}^{(\theta)}+b_{1}^{(\theta)^{2}}\right)\right\},
$$

which shows the extremal property of the hyperbolic slit mappings, provided the latter exist. Since $\phi(z)=F_{\theta}^{2}(z)-e^{2 i \theta}\left[\cos 2 \theta F_{0}^{2}(z)-i \sin 2 \theta F_{\pi / 4}^{2}(z)\right]$ clearly satisfies $\operatorname{Re}\left\{\left[i e^{-2 i \theta} F_{\theta}(z)\right]\right\}=$ const. on the boundary of $D$ and is bounded in $D$ (and zero at $z=\infty)$, we have

$$
F_{\theta}^{2}(z)=e^{2 i \theta}\left[\cos 2 \theta F_{0}^{2}(z)-i \sin 2 \theta F_{\pi / 4}^{2}(z)\right] .
$$

Hence, with the abbreviation $\gamma_{\theta}=2 b_{3}^{(\theta)}+b_{1}^{(\theta)^{2}}$,

$$
\gamma_{\theta}=e^{2 i \theta}\left[\cos 2 \theta \gamma_{0}-i \sin 2 \theta \gamma_{\pi / 4}\right] \text {, }
$$

or

(2) I owe this remark to a written communication of Professor A. C. Schaeffer. 


$$
\gamma_{\theta}=\frac{1}{2}\left(\gamma_{0}+\gamma_{\pi / 4}\right)+\frac{1}{2}\left(\gamma_{0}-\gamma_{\pi / 4}\right) e^{4 i \theta},
$$

which shows that the $\gamma_{\theta}$ are situated on the circumference of a circle.

The existence of the extremal maps can be shown by an artifice used by de Possel [3] for a similar purpose. If $D$ is simply-connected, the existence follows from the Riemann mapping theorem. If $D$ is multiply-connected, consider the extremal problem $\operatorname{Re}\left\{e^{-4 i \theta}\left(2 a_{3}+a_{1}^{2}\right)\right\}=\max$ within the family of univalent functions in $D$ which are normalized by (47). Because of the compactness of the family, the existence of an extremal function is assured. If this function, say $F(z)$, does not yield the required hyperbolic slit mapping, there is at least one boundary component, say $\Gamma$, which is not a slit of this type. Let

$$
p(w)=w+\frac{c_{1}}{w}+\frac{c_{2}}{w^{2}}+\cdots
$$

be the function mapping the simply-connected domain bounded by $\Gamma$ and containing $w=\infty$ onto the exterior of a hyperbolic slit of the desired type. If

$$
\begin{gathered}
w=F(z)=z+\frac{b_{1}}{z}+\frac{b_{2}}{z^{2}}+\cdots, \\
q(z)=p[F(z)]=z+\frac{d_{1}}{z}+\frac{d_{2}}{z^{2}}+\cdots,
\end{gathered}
$$

it is easily confirmed that $2 d_{3}+d_{1}^{2}=2 b_{3}+b_{1}^{2}+2 c_{3}+\underset{c_{1}}{2}$. Since $q(z)$ is univalent in $D$, it follows from the extremal property of $F(z)$ that

$$
\operatorname{Re}\left\{e^{-4 i \theta}\left(2 c_{3}+c_{1}^{2}\right)\right\} \leqq 0 .
$$

But this is impossible since, by the extremal property of the function $p(z)$ as applied to the competing function $p_{1}(w)=w$,

$$
\operatorname{Re}\left\{e^{-4 i \theta}\left(2 c_{3}+c_{1}^{2}\right)\right\}>0 .
$$

5. Mutually disjoint domains. 1. An interesting set of inequalities is obtained when the procedure leading to Theorem I is generalized to the case of a domain $D$ which contains a number of mutually disjoint subdomains $D_{1}, \cdots, D_{n}$. We now have to introduce a number of singularity functions $S_{\nu}(z), \nu=1, \cdots, n$, which are harmonic and single-valued in the closure of $D-D_{v}$. Restricting ourselves, for shorter formulation, to the case of schlicht domains, we then obtain the following result.

Theorem IV. Let $D$ be a schlicht domain and let $D_{1}, \cdots, D_{n}$ be mutually disjoint subdomains of $D$. Let $S_{\nu}(z)$ be the singularity functions defined above 
and let the functions $p_{\nu}(z)$ be such that $p_{\nu}(z)=0$ on the boundary $C_{\nu}$ of $D_{\nu}$ and that $p_{v}(z)+S_{v}(z)$ is harmonic in $D_{v}$. If $P(z)$ is a function which vanishes on the boundary $C$ of $D$ and for which $P(z)+\sum_{v=1}^{n} S_{v}(z)$ is harmonic in $D$, then

$$
\sum_{\nu=1}^{n} \int_{C_{\nu}}\left(p_{\nu}+\sum_{\mu=1}^{n} S_{\mu}\right) \frac{\partial p_{\nu}}{\partial n} d s \geqq \int_{C}\left(P+\sum_{\mu=1}^{n} S_{\mu}\right) \frac{\partial P}{\partial n} d s .
$$

There will be equality in (48) if, and only if, $P(z)$ vanishes at all points of $C_{\nu}$, $\nu=1, \cdots, n$.

The proof of (48) follows from the remark that the function $u(z)$ defined by

$$
\begin{array}{cr}
u(z)=p_{v}(z)+\sum_{\mu=1}^{n} S_{\mu}(z), & z \in D_{\nu}, \\
u(z)=\sum_{\mu=1}^{n} S_{\mu}(z), & z \in D-\sum_{\mu=1}^{n} D_{\mu},
\end{array}
$$

is continuous in $D$ and has on $C$ the same boundary values as the harmonic function $P(z)+\sum_{\mu-1}^{n} S_{\mu}(z)$. Hence, by the minimum property of the Dirichlet integral,

$$
\sum_{\nu=1}^{n}\left(p_{\nu}+S, p_{\nu}+S\right)_{D_{\nu}}+(S, S)_{D-\Sigma_{\nu=1}^{n} D \nu} \geqq(P+S, P+S)_{D},
$$

where the abbreviation

$$
S(z)=\sum_{v=1}^{n} S_{v}(z)
$$

has been used. By Green's formula, it follows that

$$
\sum_{\nu=1}^{n} \int_{C_{\nu}}\left(p_{\nu}+S\right) \frac{\partial}{\partial n}\left(p_{\nu}+S\right) d s+\int_{C-\Sigma_{\nu=1}^{n} C_{\nu}} S \frac{\partial S}{\partial n} d s \geqq \int_{C}(P+S) \frac{\partial}{\partial n}(P+S) d s,
$$

whence, by the boundary properties of $p_{\nu}$ and $P$,

$$
\sum_{v=1}^{n} \int_{C_{v}}\left(p_{v}+S\right) \frac{\partial p_{v}}{\partial n} d s \geqq \int_{C}(P+S) \frac{\partial P}{\partial n} d s .
$$

In view of (51), this proves (48).

Equality in (50) is possible only if the function (49) coincides with $P+S$ throughout $D$. But this means that $p_{\nu} \equiv P$ in each $D_{\nu}$, which shows that $P$ must vanish on $C_{\nu}$. Incidentally, it is also clear that the domains $D_{\nu}$ must in this case fill the entire domain $D$, since otherwise there would be regions in which $P$ is harmonic and on whose boundaries $P$ vanishes.

2. As a first application of Theorem IV, consider the case $S_{\nu}(z)$ $=\alpha_{\nu} \log \left|z-\zeta_{\nu}\right|$, where $\zeta_{\nu}$ is a point of $D_{\nu}$ and $\alpha_{\nu}$ is an arbitrary real constant. 
If $g_{\nu}\left(z, \zeta_{\nu}\right)=-\log \left|z-\zeta_{\nu}\right|+h_{\nu}\left(z, \zeta_{\nu}\right)$ and $G\left(z, \zeta_{\nu}\right)=-\log \left|z-\zeta_{\nu}\right|+H\left(z, \zeta_{\nu}\right)$ are the Green's functions of $D_{\nu}$ and $D$, respectively, we clearly have $p_{\nu}(z)$ $=\alpha_{\nu} g_{\nu}\left(z, \zeta_{\nu}\right), P(z)=\sum_{\nu=1}^{n} \alpha_{\nu} G\left(z, \zeta_{\nu}\right)$, and (48) yields

$$
\begin{aligned}
\sum_{\nu=1}^{n} \int_{C_{\nu}}\left[\alpha_{\nu} h_{\nu}\left(z, \zeta_{\nu}\right)+\sum_{\mu \neq \nu} \alpha_{\mu} \log \left|z-\zeta_{\mu}\right|\right] \alpha_{\nu} \frac{\partial g_{\nu}\left(z, \zeta_{\nu}\right)}{\partial n} d s \\
\geqq \int_{C}\left[\sum_{\nu=1}^{n} \alpha_{\nu} H\left(z, \zeta_{\nu}\right)\right] \frac{\partial}{\partial n}\left[\sum_{\nu=1}^{n} \alpha_{\nu} G\left(z, \zeta_{\nu}\right)\right] d s .
\end{aligned}
$$

Evaluating the integrals by Green's formula, we obtain

$$
\sum_{\nu=1}^{n} \alpha_{\nu}^{2} h_{\nu}\left(\zeta_{\nu}, \zeta_{\nu}\right)+2 \sum_{\nu<\mu} \alpha_{\nu} \alpha_{\mu} \log \left|\zeta_{\nu}-\zeta_{\mu}\right| \leqq \sum_{\nu, \mu=1}^{n} \alpha_{\nu} \alpha_{\mu} H\left(\zeta_{\nu}, \zeta_{\mu}\right)
$$

For $n=2,(52)$ yields the interesting discriminant inequality

$G^{2}\left(\zeta_{1}, \zeta_{2}\right) \leqq\left[H\left(\zeta_{1}, \zeta_{1}\right)-h_{1}\left(\zeta_{1}, \zeta_{1}\right)\right]\left[H\left(\zeta_{2}, \zeta_{2}\right)-h_{2}\left(\zeta_{2}, \zeta_{2}\right)\right]$.

3. Applying (52) to a problem in the theory of bounded functions, we set $n=2, \alpha_{1}=1, \alpha_{2}=-1$ and take $D$ to be the unit circle. Since, in this case, $H(\zeta, \eta)=\log \left|1-\zeta \eta^{*}\right|,(52)$ takes the form

$$
h_{1}\left(\zeta_{1}, \zeta_{1}\right)+h_{2}\left(\zeta_{2}, \zeta_{2}\right)-2 \log \left|\zeta_{1}-\zeta_{2}\right| \leqq \log \frac{\left(1-\left|\zeta_{1}\right|^{2}\right)\left(1-\left|\zeta_{2}\right|^{2}\right)}{\left|1-\zeta_{1} \zeta_{2}^{*}\right|^{2}}
$$

If the functions $f(z)$ and $g(z)$ map the unit circle onto the domains $D_{1}$ and $D_{2}$, respectively, and we set $f(z)=\zeta_{1}, g(w)=\zeta_{2}$, we have

$$
h_{1}\left(\zeta_{1}, \zeta_{1}\right)=\log \left[\left|f^{\prime}(z)\right|\left(1-|z|^{2}\right)\right], h_{2}\left(\zeta_{2}, \zeta_{2}\right)=\log \left[\left|g^{\prime}(w)\right|\left(1-|w|^{2}\right)\right] .
$$

Inserting these expressions in (53), we obtain the following result.

Let $f(z)$ and $g(z)$ be univalent and bounded by unity in $|z|<1$ and let $f(z) \neq g(w)$, where $z$ and $w$ are any two points in the unit circle. Then

$$
\left|f^{\prime}(z) g^{\prime}(w)\right| \leqq\left(\frac{1-|f(z)|^{2}}{1-|z|^{2}}\right)\left(\frac{1-|g(w)|^{2}}{1-|w|^{2}}\right)\left|\frac{f(z)-g(w)}{1-g^{*}(w) f(z)}\right|^{2} .
$$

This inequality is sharp and, for two given points $z=z_{0}$ and $w=w_{0}$, equality will hold only if the conformal maps yielded by the two functions partition the unit circle along $a$ circular arc which is orthogonal to $|z|=1$ and with respect to which $f\left(z_{0}\right)$ and $g\left(w_{0}\right)$ are inverse points.

That we have indeed equality in this case follows from the remark that, by the Schwarz inversion principle, the analytic continuation of the Green's function of $D_{1}$ across the dividing circular arc coincides with the negative value of the Green's function of $D_{2}$.

From (54) we can deduce the following result.

If $f(z)$ and $g(z)$ are univalent and bounded by unity in $|z|<1$ and if $f(z)$ 
$\neq g(w)$, where $z$ and $w$ are any two points in the unit circle, then

$$
\left|f^{\prime}(0) g^{\prime}(0)\right| \leqq 1 / 4 \text {. }
$$

This inequality is sharp and the sign of equality will occur only in the case in which $f(0)=0, g(0)=2^{-1 / 2}$ (or vice versa) and the two mappings partition the unit circle along an arc of $|z-2 g(0)|=1$.

In view of (54), (55) will be established if, for any two numbers $a, b$ such that $0<a<1,0<b<1$, it can be shown that

$$
\left(1-a^{2}\right)\left(1-b^{2}\right)\left(\frac{a+b}{1+a b}\right)^{2} \leqq \frac{1}{4} .
$$

Writing $a=\tanh \alpha, b=\tanh \beta$, we have

$$
\begin{aligned}
\left(1-a^{2}\right)\left(1-b^{2}\right)\left(\frac{a+b}{1+a b}\right)^{2} & =\left(\frac{\tanh (\alpha+\beta)}{\cosh \alpha \cosh \beta}\right)^{2} \\
& \leqq\left(\frac{\tanh (\alpha+\beta)}{\cosh \alpha \cosh \beta-\sinh \alpha \sinh \beta}\right)^{2} \\
& =\left(\frac{\sinh (\alpha+\beta)}{1+\sinh ^{2}(\alpha+\beta)}\right)^{2} \leqq \frac{1}{4}
\end{aligned}
$$

with equality if $\sinh (\alpha+\beta)=1$ and $\alpha=0$ or $\beta=0$. Choosing $\alpha=0$, we shall thus have equality for $a=0, b=2^{-1 / 2}$. The description of the extremal mappings follows by specialization of the preceding result.

4. As another application of (54), we consider a function $F(z)$ which is bounded and univalent in $|z|<1$ and does not vanish there. The functions $(F(z))^{1 / 2}$ and $-(F(z))^{1 / 2}$ are also univalent in $|z|<1$ and clearly take no common values. We may thus identify $(F(z))^{1 / 2}$ and $-(F(z))^{1 / 2}$ with, respectively, the functions $f(z)$ and $g(z)$ in (54). Hence:

If $F(z)$ is univalent, bounded by unity and $\neq 0$ in $|z|<1$, and if $z$, w are any two points in the unit circle, then

$$
\left.\left|\frac{F^{\prime}(z) F^{\prime}(w)}{(F(z) F(w))^{1 / 2}}\right| \leqq 4\left(\frac{1-|F(z)|}{1-|z|^{2}}\right)\left(\frac{1-|F(w)|}{1-|w|^{2}}\right)^{\prime}\right]\left.\frac{(F(z))^{1 / 2}+(F(w))^{1 / 2}}{1+\left(F^{*}(z) F(w)\right)^{1 / 2}}\right|^{2},
$$

with equality only if $F(z)$ maps $|z|<1$ onto the unit circle minus a rectilinear slit along an entire radius.

5. By different choices of the singularity functions $S_{\nu}$ in Theorem IV it is possible to derive a great many inequalities concerning the conformal mapping of a number of mutually disjoint domains all of which are contained in a given large domain. Although many of these inequalities are of interest in themselves, their derivations all follow the same pattern and no new ideas are involved. However, a different type of inequality is obtained if we combine the results contained in Theorems III and IV. In the interest of 
more concise formulation we again restrict ourselves to the case of schlicht domains. The modifications which are necessary if the schlicht plane is replaced by a closed Riemann surface of finite genus are quite obvious.

Theorem V. Let $D_{1}, \cdots, D_{n}$ be mutually disjoint domains in the schlicht plane and let $S_{1}(z), \cdots, S_{n}(z)$ be functions such that $\sum_{\nu=1}^{n} S_{\nu}(z)$ is harmonic and single-valued in the complement of $\sum_{\nu=1}^{n} D_{\nu}$. If $p_{\nu}(z)$ is such that $p_{\nu}(z)=0$ on the boundary $C_{\nu}$ of $D_{\nu}$ and $p_{\nu}(z)+S_{\nu}(z)$ is harmonic and single-valued in $D_{\nu}$, then

$$
\sum_{\nu=1}^{n} \int_{C_{\nu}}\left(p_{\nu}+\sum_{\mu=1}^{n} S_{\mu}\right) \frac{\partial p_{\nu}}{\partial n} d s \geqq 0 .
$$

There will be equality in (56) if, and only if, the domains $D_{\nu}$ and their boundaries cover the entire plane and if all boundaries consist of arcs satisfying $\sum_{\nu=1}^{n} S_{\nu}(z)$ $=0$.

As a first application, take $S_{\nu}(z)=\alpha_{\nu} \log \left|z-\zeta_{\nu}\right|$, where $\zeta_{\nu}$ is a finite point of $D_{\nu}$ and the real constants $\alpha_{\nu}$ satisfy $\sum_{\nu=1}^{n} \alpha_{\nu}=0$ and are otherwise arbitrary; because of the condition restricting the $\alpha_{\nu}, \sum_{\nu=1}^{n} \alpha_{\nu} \log \left|z-\zeta_{\nu}\right|$ will be regular at $z=\infty$. If $g_{\nu}\left(z, \zeta_{\nu}\right)=-\log \left|z-\zeta_{\nu}\right|+h_{\nu}\left(z, \zeta_{\nu}\right)$ is the Green's function of $D_{\nu}$, we have $p_{\nu}(z)=\alpha_{\nu} g_{\nu}\left(z, \zeta_{\nu}\right)$. Inserting this in (56) and using Green's formula, we obtain

$$
\sum_{\nu=1}^{n} \alpha_{\nu}^{2} h_{\nu}\left(\zeta_{\nu}, \zeta_{\nu}\right)+2 \sum_{\nu<\mu} \alpha_{\nu} \alpha_{\mu} \log \left|\zeta_{\nu}-\zeta_{\mu}\right| \leqq 0, \quad \sum_{\nu=1}^{n} \alpha_{\nu}=0
$$

with equality only in the case in which the entire plane is divided up into the domains $D$, by the curves $\sum_{v=1}^{n} \alpha_{v} \log \left|z-\zeta_{\nu}\right|$.

6. (57) gives rise to a number of curious geometric results. Set $n=2 m$, and divide the $\zeta_{\nu}$ into two groups of $m$ points each, for which the $\alpha_{\nu}$ are taken as 1 and -1 , respectively. Next, assume that all domains are simply-connected and geometrically congruent to each other, and that the singularities of the Green's functions all have the same relative position within the domains in question. In this case we have $h_{\nu}\left(\zeta_{v}, \zeta_{v}\right)=\log R(a)$, where $R(a)$ is the inner conformal radius [10] of the domain with respect to the point selected, and (57) leads to the following result:

Let $D$ be a simply-connected domain and let a be a fixed point of $D$. If $2 m$ replicas of $D$ are placed in the plane in such a way that no two of them overlap and if $\zeta_{1}, \cdots, \zeta_{m}, \eta_{1}, \cdots, \eta_{m}$ denote the positions of the point a in the plane,

$$
R(a) \leqq\left(\frac{\prod_{\nu, \mu=1}^{m}\left|\zeta_{\nu}-\eta_{\mu}\right|}{\prod_{\nu<\mu}\left|\zeta_{\nu}-\zeta_{\mu}\right| \prod_{\nu<\mu}\left|\eta_{\nu}-\eta_{\mu}\right|}\right)^{1 / m}
$$

where $R(a)$ is the inner conformal radius of $D$ with respect to $a$. 
For a simple domain $D, R(a)$ can be expressed in terms of the geometric constants involved and (58) becomes a purely geometric statement. If, for instance, $D$ is a circle and $a$ its center, $R(a)$ is the radius of the circle, and the right-hand side of (58) provides an upper bound for the size of the latter. From the way (58) was derived it is, however, clear that this will not be a sharp bound for the radius (an example shows, incidentally, that it cannot be more than about 8 per cent higher than the true value).

To find the domains $D$ for which (58) will be sharp, we observe from Theorem $\mathrm{V}$ that this is possible only if the entire plane can be simply covered by replicas of $D$. Furthermore, the Green's functions of the domains with $\alpha_{\nu}=1$ and the negative values of the Green's functions of the domains with $\alpha_{\nu}=-1$ must all be analytic continuations of each other. Except for the trivial case in which $D$ is a sector of angle $\pi / m$, this leaves three possibilities. $D$ may be a rectangle, an equilateral triangle, or an infinite parallel strip, and the distribution of the values \pm 1 for $\alpha_{\nu}$ must be such that across each boundary line two different values of $\alpha_{\nu}$ face each other. For squares, we thus have the following sharp result.

Let $\zeta_{1} \cdots, \zeta_{m}, \eta_{1} \cdots, \eta_{m}$ be distinct points in the plane and draw, with these points as centers, equally-sized squares of arbitrary orientation which do not overlap. If $b$ is the side of the square, then

$$
b \leqq \frac{\Gamma^{2}(1 / 4)}{4 \pi^{1 / 2}} P=1.8541 \cdots P,
$$

where $P$ is the right-hand side of (58). The constant in (59) is the best possible.

Equality in (59) is, of course, excluded unless there is an infinity of squares covering the plane in chess-board fashion. How fast equality is approached if the number of squares grows is indicated by the fact that, for a chess-board arrangement of $4 \times 4$ squares, we obtain $b=1.6469 P$. If we use the known value of the inner radius of an equilateral triangle of side $b$, we obtain the sharp inequality

$$
\left.b \leqq) \Gamma^{3}(1 / 3) / 2 \pi\right) P,
$$

analogous to (59).

Using parallel strips of width $b$, we obtain the following result.

If $2 m$ nonoverlapping linear segments of length $b$ and centers $\zeta_{1}, \cdots, \zeta_{m}$, $\eta_{1}, \cdots, \eta_{m}$ lie on the same straight line, then $b \leqq(\pi / 2) P$, where $P$ is the righthand side of (58). The constant $\pi / 2$ is the best possible.

By generalizing Theorem $\mathrm{V}$ to harmonic functions in three dimensions, similar three-dimensional results can be obtained. As an example, we mention the following inequality.

Consider $2 m$ nonoverlapping equal cubes in space whose centers are at the points $P_{1}, \cdots, P_{m}, Q_{1}, \cdots, Q_{m}$. If $b$ is the side of the cube, we have the sharp inequality 


$$
b \leqq \frac{1}{m \gamma}\left(\sum_{\nu, \mu=1}^{m} \frac{1}{\overline{P_{\nu} Q_{\mu}}}-\sum_{\nu<\mu} \frac{1}{\overline{P_{\nu} P_{\mu}}}-\sum_{\nu<\mu} \frac{1}{\overline{Q_{\nu} Q_{\mu}}}\right),
$$

where $\gamma=\lim _{P \rightarrow Q} g(P, Q)-(\overline{P Q})^{-1}$ and $g(P, Q)$ is the Green's function of the unit cube with respect to its center.

7. Passing now to function-theoretical applications of Theorem $\mathrm{V}$, we first consider the special case $n=2, \alpha_{1}=1, \alpha_{2}=-1$ of inequality (57), that is,

$$
h_{1}\left(\zeta_{1}, \zeta_{1}\right)+h_{2}\left(\zeta_{2}, \zeta_{2}\right) \leqq 2 \log \left|\zeta_{1}-\zeta_{2}\right| .
$$

If $D_{1}$ and $D_{2}$ are simply-connected and we introduce the functions $f(z)$ and $g(z)$ which map the interior of the unit circle onto $D_{1}$ and $D_{2}$, respectively, (60) can be expressed as the following result concerning these two functions.

If $f(z)$ and $g(z)$ are univalent for $|z|<1$ and if $f(z) \neq g(w)$, where $z$ and $w$ are two arbitrary points in the unit circle, then

$$
\frac{\left|f^{\prime}(z) g^{\prime}(w)\right|}{|f(z)-g(w)|^{2}} \leqq \frac{1}{\left(1-|z|^{2}\right)\left(1-|w|^{2}\right)},
$$

with equality only in the case in which the two functions map the unit circle onto complementary half-planes, with respect to whose dividing line the points $f(z)$ and $g(w)$ are symmetric.

(61) can be made to yield some information on univalent functions in the unit circle. If $F(z)$ is regular and univalent and does not vanish in $|z|<1$, both $(F(z))^{1 / 2}$ and $-(F(z))^{1 / 2}$ are univalent in the unit circle and they do not take common values. They may thus be identified with the functions $f(z)$ and $g(z)$ in (61). This yields

$$
\left|\frac{F^{\prime}(z)}{F(z)}\right| \leqq \frac{4}{1-|z|^{2}}
$$

which, incidentally, reduces to the Koebe $1 / 4$-theorem for $z=0$. Integrating (62) along a radial path from 0 to $z$, we obtain

$$
\left|\log \frac{F(z)}{F(0)}\right| \leqq 2 \log \frac{1+|z|}{1-|z|},
$$

whence

$$
|F(0)|\left(\frac{1-|z|}{1+|z|}\right)^{2} \leqq|F(z)| \leqq|F(0)|\left(\frac{1+|z|}{1-|z|}\right)^{2}
$$

and the information that

$$
\operatorname{Re}\left\{\frac{F(z)}{F(0)}\right\}>0 \text { for }|z|<\tanh \frac{\pi}{8} .
$$

6. Miscellaneous problems. 1 . The first result of this section concerns a 
sharpening of Theorem I which is possible if the singularity function $S(z)$ satisfies

$$
\int_{C} \frac{\partial S(z)}{\partial n} d s \neq 0
$$

Theorem I can be replaced by the more precise inequality

$$
\int_{C} S \frac{\partial p}{\partial n} d s \geqq \int_{C_{1}} S \frac{\partial p_{1}}{\partial n} d s+\left[(\omega, \omega)_{D_{1}-D}\right]^{-1}\left(\int_{C} \frac{\partial S}{\partial n} d s\right)^{2},
$$

where $\omega(z)$ denotes the harmonic function which has the boundary values 1 and 0 on $C$ and $C_{1}$, respectively.

To prove (64), we consider the function $u(z)$ defined by $u(z)=p(z)+S(z)+\alpha, \quad z \in D, \quad u(z)=S(z)+\alpha \omega(z), \quad z \in D_{1}-D$, where $\alpha$ is an arbitrary real constant. Since $u(z)$ is continuous in $D_{1}$ and has on $C_{1}$ the same boundary values as $p_{1}(z)+S(z)$, it follows from the minimum property of the Dirichlet integral that

$$
(p+S, p+S)_{D}+(S+\alpha \omega, S+\omega)_{D_{1}-D} \geqq\left(p_{1}+S, p_{1}+S\right)_{D_{1}} .
$$

Transforming this inequality as in the proof of Theorem I, we find

$$
\int_{C} S \frac{\partial p}{\partial n} d s+2 \alpha(S, \omega)_{D_{1}-D}+\alpha^{2}(\omega, \omega)_{D_{1}-D} \geqq \int_{C_{1}} S \frac{\partial p_{1}}{\partial n} d s .
$$

The best inequality of this type is obtained for

$$
\alpha=-\frac{(S, \omega)_{D_{1}-D}}{(\omega, \omega)_{D_{1}-D}}
$$

If we choose this value of $\alpha$ and observe that, in view of the boundary properties of $\omega(z)$,

$$
(S, \omega)_{D_{1}-D}=\int_{C} \frac{\partial S}{\partial n} d S
$$

we obtain (64).

2. If both $D$ and $D_{1}$ are simply-connected, $D_{1}-D$ is doubly-connected and $(\omega, \omega)_{D_{1}-D}$ can be expressed in terms of the Riemann modulus $M$ of this domain. We have

$$
(\omega, \omega)_{D_{1}-D}=\frac{2 \pi}{\log M}
$$

which is easily confirmed by evaluating the integral for a circular ring and by 
observing that the Dirichlet integral is invariant under a conformal mapping. If we further set $S(z)=\sum_{v=1}^{n} \alpha_{\nu} \log \left|z-\zeta_{\nu 1}\right|$, where the $\zeta_{\nu}$ are points of $D$ and the $\alpha_{\nu}$ are arbitrary real constants, we have $p(z)=\sum_{\nu=1}^{n} \alpha_{\nu} g\left(z, \zeta_{\nu}\right)$, where $g\left(z, \zeta_{\nu}\right)=-\log \left|z-\zeta_{\nu}\right|+h\left(z, \zeta_{\nu}\right)$ is the Green's function of $D$. Inserting this in (64), we arrive after some manipulation at the inequality

$$
\sum_{\nu, \mu=1}^{n} \alpha_{\nu} \alpha_{\mu} h\left(\zeta_{\nu}, \zeta_{\mu}\right) \leqq \sum_{\nu, \mu=1}^{n} \alpha_{\nu} \alpha_{\mu} h_{1}\left(\zeta_{\nu}, \zeta_{\mu}\right)-\left(\sum_{\nu=1}^{n} \alpha_{\nu}\right)^{2} \log M .
$$

For $n=1, \alpha_{1}=1$, this reduces to

$$
h(\zeta, \zeta) \leqq h_{1}(\zeta, \zeta)-\log M .
$$

If $D$ is finite and $r(\zeta)$ is the inner conformal radius of $D$ [10], we have $h(\zeta, \zeta)=\log r(\zeta) .\left(65^{\prime}\right)$ is thus equivalent to the following result.

Let $D$ and $D_{1}$ be two finite simply-connected domains such that $D \subset D_{1}$ and let $r(\zeta)$ and $R(\zeta)$ denote the inner conformal radii of $D$ and $D_{1}$, respectively, with respect to a point $\zeta$ of $D$. If $M$ denotes the Riemann modulus of the doublyconnected domain $D_{1}-D$, then

$$
M \leqq R(\zeta) / r(\zeta) .
$$

If $\dot{D}$ and $D_{1}$ contain the point at infinity, we may set $S(z)=-\log |z|$, $p(z)=g(z, \infty)=\log |z|+h(z, \infty)$. Similarly to $(65)^{\prime}$, we obtain

$$
-h(\infty, \infty) \leqq-h_{1}(\infty)-\log M \text {. }
$$

Since $h(\infty, \infty)=-\log r$, where $r$ is the outer conformal radius [10] of the complement of $D, M$ can also be estimated in terms of the outer radii.

If the outer conformal radii of the inner and outer boundaries of a finite doubly-connected domain of modulus $M$ are $r$ and $R$, respectively, then

$$
M \leqq R / r .
$$

3. To illustrate the type of information regarding bounded conformal maps of the unit circle which can be obtained from (65), we set $n=2, \alpha_{1}=\alpha_{2}$ $=1$, and take $D_{1}$ to be the unit circle. We have $h_{1}(z, \zeta)=\log \left|1-\zeta^{*} z\right|$ and, if $f(z)$ denotes the function mapping $D$ onto the unit circle,

$$
h(z, \zeta)=\log \left[\left|\frac{z-\zeta}{f(z)-f(\zeta)}\right|\left|1-f^{*}(\zeta) f(z)\right|\right] .
$$

We further assume that $D$ contains the origin and that $f(0)=0$. Setting $\zeta=0$ and passing to the function $F$ inverse to $f$, we arrive after an elementary computation at the following result.

Let $w=F(z)$ map $|z|<1$ onto a schlicht domain $D$ contained in $|w|<1$ and let $F(0)=0$. If $M$ is the Riemann modulus of the doubly-connected domain bounded by $|w|=1$ and the boundary of $D$, then 


$$
\left|F^{\prime}(z) F^{\prime}(0)\right|\left|\frac{F(z)}{z}\right|^{2} \leqq M^{-4}\left(\frac{1-|F(z)|^{2}}{1-|z|^{2}}\right),
$$

with equality in the case in which $D$ is bounded by the fourth-order curve

$$
\left|w\left(\frac{w-f(z)}{1-f^{*}(z) w}\right)\right|=\text { const. }
$$

4. We now turn to a different type of extremal problem. Let $D$ be $a$ schlicht domain of finite connectivity and let $U(s)$ denote a piecewise continuous function of $s$, where $s$ is the length-parameter along the boundary $C$ of $D$; let further $\alpha$ and $\beta$ denote two distinct points of $D$. Consider now all doublyconnected subdomains $D^{\prime}$ of $D$ which do not contain $\alpha$ and $\beta$ and whose boundaries $C+C^{\prime}$ contain $C$, and associate with $D^{\prime}$ the harmonic function $u(z)$ in $D^{\prime}$ which takes the boundary values $U(s)$ and zero on $C$ and $C^{\prime}$, respectively. With these definitions, find the domain $D^{\prime}$ for which

$$
(u, u)_{D^{\prime}}=\iint_{D^{\prime}}\left(u_{x}^{2}+u_{y}^{2}\right) d x d y=\min .
$$

We shall show that the problem (66) is solved by a domain $D_{0}^{\prime}$ whose boundary component $C^{\prime}$ is an analytic slit connecting $\alpha$ and $\beta$ and that the associated function $u_{0}(z)$ satisfies

$$
\left\{\frac{\partial u_{0}}{\partial n}\right\}_{z=z_{1}}=\left\{\frac{\partial u_{0}}{\partial_{n}}\right\}_{z=z_{2}},
$$

if $z_{1}$ and $z_{2}$ are two geometrically coinciding points on opposite edges of the slit.

We first remark that the existence of a solution to our problem follows from compactness considerations. Furthermore, an approximation argument shows that it is sufficient to establish the inequality

$$
\left(u_{0}, u_{0}\right)_{D_{0^{\prime}}} \leqq(u, u)_{D^{\prime}} \text {, }
$$

where the boundary component $C^{\prime}$ of $D^{\prime}$ consists of one or more analytic curves. If $D^{\prime}$ is of this type, we may connect $\alpha$ and $\beta$ by a continuous arc $\gamma$ which may even be assumed to be analytic - such that $\gamma$ has no points in common with $D^{\prime}$. We denote the domain bounded by $C$ and $\gamma$ by $D^{\prime \prime}$, and we extend the definition of $u(z)$ to all points of $D^{\prime \prime}$ by setting $u(z)=0$ in $D^{\prime \prime}-D^{\prime}$; obviously, this function $u(z)$ is continuous in $D^{\prime \prime}$.

We now construct a two-sheeted domain $D^{*}$ by crosswise joining two replicas of $D^{\prime \prime}$ along $\gamma$, and we extend the definition of $u(z)$ to all points of $D^{*}$ by setting $u\left(z_{1}\right)+u\left(z_{2}\right)=0$ if $z_{1}$ and $z_{2}$ are points which lie one on top of the other. Clearly, this function $u(z)$ is continuous in $D^{*}$ and it has the boundary values $U(s)$ and $-U(s)$ on $C$ and its replica, respectively. We now introduce the function $u_{0}(z)$ which has the same boundary values and is harmonic throughout $D^{*}$. By the minimum property of the Dirichlet integral, we have 


$$
\left(u_{0}, u_{0}\right)_{D^{*}} \leqq(u, u)_{D^{*}}=2(u, u)_{D^{\prime}}
$$

By symmetry, $u_{0}\left(z_{1}\right)=-u_{0}\left(z_{2}\right)$ if $z_{1}$ and $z_{2}$ are two points with the same projection onto the plane, and the curve $u_{0}(z)=0$ will therefore decompose $D^{*}$ into two identical schlicht slit-domains $D_{0}^{\prime}$. Since $\alpha$ and $\beta$ are branch points of $D^{*}$, this slit-which, since it satisfies $u(z)=0$, is obviously analytic-must connect $\alpha$ and $\beta$. It is further clear that $\left(u_{0}, u_{0}\right)_{D_{0}}=2\left(u_{0}, u_{0}\right)_{D_{0}^{\prime}}$ and this, together with (69), proves the minimum property (68) of $D_{0}^{\prime}$. That the normal derivatives of $u_{0}(z)$ satisfy the relation (67) at the points of the slit is also an immediate consequence of the fact that $u_{0}\left(z_{1}\right)=-u_{0}\left(z_{2}\right)$ if $z_{1}$ and $z_{2}$ are two points of $D^{*}$ with the same projection onto the plane.

The same method of proof will also dispose of the case in which there are $m$ pairs of points of $D$, say $\alpha_{1}, \beta_{1}, \cdots, \alpha_{m}, \beta_{m}$, which are to be outside $D^{\prime}$, and in which we prescribe, moreover, that both points of a pair are to be within the same "hole." We obtain an extremal domain which, in addition to $C$, is bounded by $m$ analytic slits each connecting the two points of a pair; the extremal function will again have the property (67).

5. (67) can be used in order to obtain explicit expressions for the extremal functions. We illustrate this in the case of the following problem: Given four distinct points $\alpha_{1}, \alpha_{2}, \alpha_{3}, \alpha_{4}$, to find two disjoint continua $C_{1}$ and $C_{2}$ containing the pairs $\alpha_{1}, \alpha_{2}$ and $\alpha_{3}, \alpha_{4}$, respectively, such that the Riemann modulus of the complement $D$ of $C_{1}+C_{2}$ is a maximum [13].

If $u(z)$ is harmonic in $D$ and takes the boundary values 0 and 1 on $C_{1}$ and $C_{2}$, respectively, the modulus $M$ of $D$ is given by $(u, u)_{D}=2 \pi / \log M$. We thus have to minimize this Dirichlet integral under the above conditions; the existence of a minimizing domain follows again from a compactness argument. If we apply our solution of the problem (66) in turn to the curves $C_{1}$ and $C_{2}$-using the functions $u(z)$ and $1-u(z)$, respectively-we find that in the extremal case both $C_{1}$ and $C_{2}$ are analytic arcs along which the extremal function satisfies the condition (67).

If $w=f(z)$ maps the extremal domain onto the circular ring $1<|w|<M$, we have $\log |f(z)|=u(z) \log M$, and thus

$$
\frac{\partial u}{\partial n}=\frac{1}{i} \frac{f^{\prime}(z)}{f(z)} \frac{d z}{d s}
$$$$
z \in C_{1}, C_{2}
$$

Since $(d z / d s)^{2}$ has the same values on opposite edges of the slits, it follows therefore from (67) that $\left(f^{\prime}(z) / f(z)\right)^{2}$ is single-valued in the entire plane. The only singularities of this function are situated at the points $\alpha_{\nu}, \nu=1,2,3,4$. In view of the mapping properties of $f(z)$, these are simple poles. Hence the function

$$
\left(\frac{f^{\prime}(z)}{f(z)}\right)^{2} \prod_{\nu=1}^{4}\left(z-\alpha_{v}\right)
$$


is regular and single-valued at all finite points of the plane. Since $f^{\prime} / f$ has a double zero at $z=\infty$, the same is also true at infinity, and the function (70) must reduce to a constant. It thus follows, in accordance with [13], that

$$
\log f(z)=c \int^{z} \frac{d z}{\left(\prod_{\nu=1}^{4}\left(z-\alpha_{v}\right)\right)^{1 / 2}} .
$$

\section{REFERENCES}

1. S. Bergman, The kernel function and conformal mapping, New York, American Mathematical Society, 1950.

2. S. Bergman and M. Schiffer, Kernel functions and conformal mapping, Compositio Math. vol. 8 (1951) pp. 205-249.

3. R. de Possel, Zum Parallelschlitztheorem unendlichvielfach zusammenhaengender Gebiete, Göttinger Nachrichten (1931) pp. 199-202.

4. P. Garabedian and M. Schiffer, Identities in the theory of conformal mapping, Trans. Amer. Math. Soc. vol. 65 (1949) pp. 187-238.

5. G. M. Golusin, Rec. Math. N.S. vol. 3 (45) (1938) pp. 321-330.

6. H. Grötzsch, Über einige Extremalprobleme der konformen Abbildung, Berichte über die Verhandlungen der Sächsischen Akademie der Wissenschaften zu Leipzig vol. 80 (1928) pp. 497-502.

7. - Über die Verzerrung bei schlichter konformer Abbildung mehrfach zusammenhängender schlichter Bereiche, Berichte über die Verhandlungen der Sächsischen Akademie der Wissenschaften zu Leipzig vol. 83 (1931) pp. 283-297.

8. H. Grunsky, Neue Abschätzungen zur Theorie der konformen Abbildung ein- und mehrfachzusammenhängender Bereiche, Schr. Math. Sem. Univ. Berlin vol. 1 (1932) pp. 95-140.

9. - Koeffizientenbedingungen für schlicht abbildende meromorphe Funktionen, Math. Zeit. vol. 45 (1939) pp. 29-61.

10. G. P6lya and G. Szegö, Isoperimetric inequalities in mathematical physics, Princeton University Press, 1951.

11. A. C. Schaeffer and D. C. Spencer, Coefficient regions for schlicht functions, New York, American Mathematical Society, 1950.

12. M. Schiffer, Sur un problème d'extrémum de la représentation conforme, Bull. Soc. Math. France vol. 66 (1938) pp. 48-55.

13. - On the modulus of a doubly-connected domain, Quart. J. Math. Oxford Ser. vol. 17 (1946) pp. 197-213.

14. - Hadamard's formula and variation of domain functions, Amer. J. Math. vol. 68 (1946) pp. 417-448.

15. - The kernel function of an orthonormal system, Duke Math. J. vol. 13 (1946) pp. 529-540.

16. M. Schiffer and G. Szegö, Virtual mass and polarization, Trans. Amer. Math. Soc. vol. 67 (1949) pp. 130-205.

17. G. Springer, The coefficient problem for schlicht mappings of the exterior of the unit circle, Trans. Amer. Math. Soc. vol. 70 (1951) pp. 421-450.

STANFORD UNIVERSITY,

Stanford, Calif.

WASHINGTON UNIVERSITY,

ST. Louis, Mo. 